\title{
Random Attractors for Stochastic Retarded Reaction-Diffusion Equations on Unbounded Domains
}

\author{
Xiaoquan Ding ${ }^{1}$ and Jifa Jiang ${ }^{2}$ \\ ${ }^{1}$ School of Mathematics and Statistics, Henan University of Science and Technology, Luoyang, Henan 471023, China \\ ${ }^{2}$ School of Mathematics and Science, Shanghai Normal University, Shanghai 200234, China \\ Correspondence should be addressed to Xiaoquan Ding; xqding@haust.edu.cn
}

Received 10 March 2013; Accepted 28 May 2013

Academic Editor: Grzegorz Lukaszewicz

Copyright (c) 2013 X. Ding and J. Jiang. This is an open access article distributed under the Creative Commons Attribution License, which permits unrestricted use, distribution, and reproduction in any medium, provided the original work is properly cited.

\begin{abstract}
This paper is devoted to a stochastic retarded reaction-diffusion equation on all $d$-dimensional space with additive white noise. We first show that the stochastic retarded reaction-diffusion equation generates a random dynamical system by transforming this stochastic equation into a random one through a tempered stationary random homeomorphism. Then, we establish the existence of a random attractor for the random equation. And the existence of a random attractor for the stochastic equation follows from the conjugation relation between two random dynamical systems. The pullback asymptotic compactness is proved by uniform estimates on solutions for large space and time variables. These estimates are obtained by a cut-off technique.
\end{abstract}

\section{Introduction}

The study of stochastic functional differential equations is motivated by the fact that, when one wants to model some evolution phenomena arising in physics, chemistry, biology, and other sciences, some hereditary characteristics such as aftereffect, time-lag, and time delay can appear in the variables. On the other hand, one of the most interesting problems concerning stochastic functional differential equations is to understood the asymptotic behavior of the solutions when time grows to infinite, since it can provide useful information about the future of the phenomenon described in the model.

It is known that the asymptotic behavior of random systems can be captured by a random attractor, which was introduced in $[1,2]$ as an extension of the attractor theory of deterministic systems in [3-5]. For stochastic PDEs without any hereditary features, the existence of random attractors has been investigated by many authors; see, for example, [6$20]$ and the references therein. However, this problem is not well studied in the case of stochastic retarded PDEs.

In this paper, we investigate the asymptotic behavior of solutions to the following stochastic retarded reaction- diffusion equation with additive noise defined in the entire space $\mathbb{R}^{d}$ :

$$
d u+(\lambda u-\Delta u) d t=\left(f\left(u^{t}\right)(x)+g(x)\right) d t+d W,
$$

where $\lambda$ is a positive constant, $g$ is a given function defined on $\mathbb{R}^{d}, f$ is a nonlinear functional satisfying certain conditions, and $W$ is a two-sided infinite dimensional Wiener process on a probability space which will be specified later.

We note that the asymptotic behavior of several deterministic retarded PDEs on bounded domains was studied in [21-25], and the case of retarded Navier-Stokes equations on some unbounded domains was treated in [26]. The random attractor for retarded stochastic differential equations was considered in [27] by monotone methods. Recently, in the case of stochastic retarded lattice dynamical systems defined on the entire integer set, the existence of a random attractor was proved in $[28,29]$. Here we prove the existence of a random attractor for the stochastic retarded reactiondiffusion equation defined in $\mathbb{R}^{d}$. It is worth mentioning that the asymptotic behavior of the nonretarded version of (1) was investigated recently in [9]. 
Notice that Sobolev embeddings are not compact when domains are unbounded. This introduces a major obstacle for proving existence of attractors for PDEs on unbounded domains. Under certain circumstances, the tail-estimates method can be used to deal with the problem caused by the unboundedness of domains. This approach was developed in $[30,31]$ for deterministic nonretarded PDEs and used in [9-11, $13,17,18]$ for stochastic systems. At the same time, the present of delays is another obstacle, which makes phase spaces not reflexive and increases the difficulty of uniform estimates. In this paper, we will develop a tail-estimates approach for stochastic retarded PDEs on unbounded domains and prove the existence of a compact random attractor for the stochastic retarded reaction-diffusion equation (1), in particular, defined on the unbounded domain $\mathbb{R}^{d}$. The idea is based on the observation that the solutions of the equation are uniformly small when space and time variables are sufficiently large. It is clear that our method can be used for a variety of other equations, as it was for the nonretarded case.

For convenience, hereafter we adopt the following notations. We denote by $\|\cdot\|$ and $(\cdot, \cdot)$ the norm and the inner product in $L^{2}\left(\mathbb{R}^{d}\right)$. Otherwise, the norm of a general Banach space $X$ is written as $\|\cdot\|_{X}$. For $\nu>0$, let $\mathscr{C}$ denote the Banach space of all continuous functions $\xi:[-\nu, 0] \rightarrow L^{2}\left(\mathbb{R}^{d}\right)$ endowed with the supremum norm $\|\xi\|_{\mathscr{C}}=\sup _{s \in[-\nu, 0]}\|\xi(s)\|$. For any real number $a \leq b, t \in[a, b]$, and any continuous function $u:[a-v, b] \rightarrow L^{2}\left(\mathbb{R}^{d}\right), u^{t}$ denotes the element of $\mathscr{C}$ given by $u^{t}(s)=u(t+s)$ for $s \in[-v, 0]$.

The rest of the paper is organized as follows. In the next section, we introduce basic concepts concerning random dynamical systems and random attractors. In Section 3, we define a continuous random dynamical system for the stochastic retarded reaction-diffusion equation on $\mathbb{R}^{d}$. The existence of the random attractor is given in Section 4.

\section{Preliminaries}

In this section, we introduce some basic concepts related to random attractors for random dynamical systems. The reader is referred to $[1,2,6,32-34]$ for more details.

Let $\left(X,\|\cdot\|_{X}\right)$ be a separable Banach space with Borel $\sigma$ algebra $\mathscr{B}(X)$ and $(\Omega, \mathscr{F}, \mathbb{P})$ a probability space.

Definition 1. $\left(\Omega, \mathscr{F}, \mathbb{P},\left(\vartheta_{t}\right)_{t \in \mathbb{R}}\right)$ is called a metric dynamical system if $\vartheta: \mathbb{R} \times \Omega \rightarrow \Omega$ is $(\mathscr{B}(\mathbb{R}) \otimes \mathscr{F}, \mathscr{F})$-measurable, and $\vartheta_{0}$ is the identity on $\Omega, \vartheta_{s+t}=\vartheta_{t} \circ \vartheta_{s}$ for all $s, t \in \mathbb{R}$ and $\vartheta_{t} \mathbb{P}=\mathbb{P}$ for all $t \in \mathbb{R}$.

Definition 2. A set $A \subset \Omega$ is called invariant with respect to $\left(\vartheta_{t}\right)_{t \in \mathbb{R}}$, if, for all $t \in \mathbb{R}$, it holds that

$$
\vartheta_{t}^{-1} A=A
$$

Definition 3. A continuous random dynamical system on $X$ over a metric dynamical system $\left(\Omega, \mathscr{F}, \mathbb{P},\left(\vartheta_{t}\right)_{t \in \mathbb{R}}\right)$ is a mapping

$$
\varphi: \mathbb{R}^{+} \times \Omega \times X \longrightarrow X, \quad(t, \omega, x) \longrightarrow \varphi(t, \omega, x),
$$

which is $\left(\mathscr{B}\left(\mathbb{R}^{+}\right) \otimes \mathscr{F} \otimes \mathscr{B}(X), \mathscr{B}(X)\right)$-measurable, and, for all $\omega \in \Omega$,

(i) $\varphi(t, \omega, \cdot): X \rightarrow X$ is continuous for all $t \in \mathbb{R}^{+}$;

(ii) $\varphi(0, \omega, \cdot)$ is the identity on $X$;

(iii) $\varphi(t+s, \omega, \cdot)=\varphi\left(t, \vartheta_{s} \omega, \cdot\right) \circ \varphi(s, \omega, \cdot)$ for all $s, t \in \mathbb{R}^{+}$.

Definition 4. A random set $D$ is a multivalued mapping $D$ : $\Omega \rightarrow \mathscr{B}(X)$ such that, for every $x \in X$, the mapping $\omega \rightarrow d(x, D(\omega))$ is measurable, where $d(x, B)$ is the distance between the element $x$ and the set $B \subset X$. It is said that the random set is bounded (resp., closed or compact) if $D(\omega)$ is bounded (resp., closed or compact) for $\mathbb{P}$ a.e. $\omega \in \Omega$.

Definition 5. A random variable $r: \Omega \rightarrow(0, \infty)$ is called tempered with respect to $\left(\vartheta_{t}\right)_{t \in \mathbb{R}}$, if for $\mathbb{P}$ a.e. $\omega \in \Omega$

$$
\lim _{t \rightarrow \infty} e^{-\beta t} r\left(\vartheta_{-t} \omega\right)=0 \quad \forall \beta>0 .
$$

A random set $D$ is called tempered if $D(\omega)$ is contained in a ball with center zero and tempered radius $r(\omega)$ for all $\omega \in \Omega$.

Remark 6. If $r>0$ is tempered, then

(1) for any $\tau \in \mathbb{R}, r\left(\vartheta_{\tau} \cdot\right)$ is tempered;

(2) for any $\alpha>0$ and $\mathbb{P}$ a.e. $\omega \in \Omega$

$$
R(\omega)=\int_{-\infty}^{0} e^{\alpha s} r\left(\vartheta_{s} \omega\right) d s<\infty,
$$

and $R$ is tempered.

Moreover, if, for $\mathbb{P}$ a.e. $\omega \in \Omega, r\left(\vartheta_{t} \omega\right)$ is continuous in $t$, then $R\left(\vartheta_{t} \omega\right)$ is continuous in $t$ for such $\omega$, and, for any $\nu>0$, $\sup _{\sigma \in[-\nu, 0]} r\left(\vartheta_{\sigma} \cdot\right)$ is tempered.

Hereafter, we always assume that $\varphi$ is a continuous random dynamical system over $\left(\Omega, \mathscr{F}, \mathbb{P},\left(\vartheta_{t}\right)_{t \in \mathbb{R}}\right)$, and $\mathscr{D}$ is a collection of random subsets of $X$.

Definition 7. A random set $K$ is called a random absorbing set in $\mathscr{D}$ if, for every $B \in \mathscr{D}$ and $\mathbb{P}$ a.e. $\omega \in \Omega$, there exists $t_{B}(\omega)>0$ such that

$$
\varphi\left(t, \vartheta_{-t} \omega, B\left(\vartheta_{-t} \omega\right)\right) \subseteq K(\omega) \quad \forall t \geq t_{B}(\omega)
$$

Definition 8. A random set $\mathscr{A}$ is called a $\mathscr{D}$-random attractor (D-pullback attractor) for $\varphi$ if the following hold:

(i) $\mathscr{A}$ is a random compact set;

(ii) $\mathscr{A}$ is strictly invariant; that is, for $\mathbb{P}$ a.e. $\omega \in \Omega$ and all $t \geq 0$,

$$
\varphi(t, \omega, \mathscr{A}(\omega))=\mathscr{A}\left(\vartheta_{t} \omega\right)
$$

(iii) $\mathscr{A}$ attracts all sets in $\mathscr{D}$; that is, for all $B \in \mathscr{D}$ and $\mathbb{P}$ a.e. $\omega \in \Omega$,

$$
\lim _{t \rightarrow \infty} d\left(\varphi\left(t, \vartheta_{-t} \omega, B\left(\vartheta_{-t} \omega\right)\right), \mathscr{A}(\omega)\right)=0
$$


where $d$ is the Hausdorff semimetric given by $d(E, F)=\sup _{x \in E} \inf _{y \in F}\|x-y\|_{X}$ for any $E \subseteq X$ and $F \subseteq X$. [33].

We remark that if $\mathscr{A} \in \mathscr{D}$, then this attractor is unique

Definition 9. $\varphi$ is said to be $\mathscr{D}$-pullback asymptotically compact in $X$ if, for all $B \in \mathscr{D}$ and $\mathbb{P}$ a.e. $\omega \in \Omega,\left\{\varphi\left(t_{n}\right.\right.$, $\left.\left.\vartheta_{-t_{n}} \omega, x_{n}\right)\right\}_{n=1}^{\infty}$ has a convergent subsequence in $X$ whenever $t_{n} \rightarrow \infty$, and $x_{n} \in B\left(\vartheta_{-t_{n}} \omega\right)$.

The following existence result on a random attractor for a continuous random dynamical system can be found in [2, 34]. First, recall that a collection $\mathscr{D}$ of random subsets of $X$ is called inclusion closed if whenever $E$ is an arbitrary random set and $F$ is in $\mathscr{D}$ with $E(\omega) \subset F(\omega)$ for $\mathbb{P}$ a.e. $\omega \in \Omega$, then $E$ must belong to $\mathscr{D}$.

Proposition 10. Let $\mathscr{D}$ be an inclusion-closed collection of random subsets of $X$ and $\varphi$ a continuous random dynamical system on $X$ over $\left(\Omega, \mathscr{F}, \mathbb{P},\left(\vartheta_{t}\right)_{t \in \mathbb{R}}\right)$. Suppose that $K \in \mathscr{D}$ is a closed random absorbing set for $\varphi$ in $\mathscr{D}$ and $\varphi$ is $\mathscr{D}$-pullback asymptotically compact in $X$. Then $\varphi$ has a unique $\mathscr{D}$-random attractor $\mathscr{A}$ which is given by

$$
\mathscr{A}(\omega)=\bigcap_{\tau \geq 0} \varphi\left(t, \vartheta_{t \geq \tau} \omega, K\left(\vartheta_{-t}\right)\right) .
$$

In this paper, we will take $\mathscr{D}$ as the collection of all tempered random subsets of $\mathscr{C}$ and prove the stochastic retarded reaction-diffusion equation on $\mathbb{R}^{d}$ has a $\mathscr{D}$-random attractor.

\section{Stochastic Retarded Reaction-Diffusion Equations on $\mathbb{R}^{d}$ with Additive Noise}

In this section, we show that there is a continuous random dynamical system generated by the stochastic retarded reaction-diffusion equation on $\mathbb{R}^{d}$ with additive white noise:

$$
\begin{array}{r}
d u+(\lambda u-\Delta u) d t=\left(f\left(u^{t}\right)(x)+g(x)\right) d t+d W, \\
x \in \mathbb{R}^{d}, \quad t>0,
\end{array}
$$

with the initial condition

$$
u(t, x)=u^{0}(t, x), \quad x \in \mathbb{R}^{d}, t \in[-v, 0] .
$$

Here $\lambda$ is a positive constant, $g$ is a given function in $L^{2}\left(\mathbb{R}^{d}\right)$, $W$ is an $L^{2}\left(\mathbb{R}^{d}\right)$-valued two-sided Wiener process with a symmetric nonnegative finite trace covariance operator $Q$ defined on a probability space which will be specified below, and $f: \mathscr{C} \rightarrow L^{2}\left(\mathbb{R}^{d}\right)$ is a continuous mapping satisfying the following conditions:

$\left(\mathrm{A}_{1}\right) f(0)=0$;

$\left(\mathrm{A}_{2}\right)$ there exists a positive continuous function $l_{f}(r)$ with

$$
\lim _{r \rightarrow \infty} \frac{l_{f}(r)}{r^{k_{0}}}=0
$$

for some positive integer $k_{0}$ such that, for all $\xi, \eta \in \mathscr{C}$ with $\|\xi\| \leq r$ and $\|\eta\| \leq r$,

$$
\|f(\xi)-f(\eta)\| \leq l_{f}(r)\|\xi-\eta\|_{\mathscr{C}}
$$

$\left(\mathrm{A}_{3}\right)$ there exist positive constants $\alpha_{0}$ and $c_{f}$ such that, for all $\alpha \in\left(0, \alpha_{0}\right), t>0, u \in C\left([-v, t] ; L^{2}\left(\mathbb{R}^{d}\right)\right)$, and $x \in \mathbb{R}^{d}$,

$$
\int_{0}^{t} e^{\alpha s}\left|f\left(u^{s}\right)(x)\right|^{2} d s \leq c_{f}^{2} \int_{-\nu}^{t} e^{\alpha s}|u(s)(x)|^{2} d s
$$

$\left(\mathrm{A}_{4}\right) \lambda>c_{f}$

In the sequel, we consider the probability space $(\Omega, \mathscr{F}, \mathbb{P})$ where

$$
\Omega=\left\{\omega \in C\left(\mathbb{R}, L^{2}\left(\mathbb{R}^{d}\right)\right): \omega(0)=0\right\},
$$

$\mathscr{F}$ is the Borel $\sigma$-algebra induced by the compact-open topology of $\Omega$, and $\mathbb{P}$ the corresponding Wiener measure on $(\Omega, \mathscr{F})$ with respect to the covariance operator $Q$. Let

$$
\vartheta_{t} \omega(\cdot)=\omega(\cdot+t)-\omega(t), \quad t \in \mathbb{R} .
$$

Then $\left(\Omega, \mathscr{F}, \mathbb{P},\left(\vartheta_{t}\right)_{t \in \mathbb{R}}\right)$ is an ergodic metric dynamical system. Since the above probability space is canonical, we have

$$
W(t, \omega)=\omega(t), \quad W\left(t, \vartheta_{s} \omega\right)=W(t+s, \omega)-W(s, \omega) .
$$

Similar to Proposition A.1 in [34], we can find that there exists a full $\mathbb{P}$-measure $\left\{\vartheta_{t}\right\}_{t \in \mathbb{R}}$-invariant set $\widetilde{\Omega} \in \mathscr{F}$ such that for each $\omega \in \widetilde{\Omega}$

$$
\lim _{t \rightarrow \pm \infty} \frac{\|W(t, \omega)\|}{t}=0
$$

Let $\overline{\mathscr{F}}$ be the $\mathbb{P}$-completion of $\mathscr{F}$, and let

$$
\mathscr{F}_{t}=\bigvee_{s \leq t} \mathscr{F}_{s}^{t}, \quad t \in \mathbb{R}
$$

with

$$
\mathscr{F}_{s}^{t}=\sigma\left\{W\left(\tau_{2}\right)-W\left(\tau_{1}\right): s \leq \tau_{1} \leq \tau_{2} \leq t\right\} \vee \mathscr{N},
$$

where $\sigma\left\{W\left(\tau_{2}\right)-W\left(\tau_{1}\right): s \leq \tau_{1} \leq \tau_{2} \leq t\right\}$ is the smallest $\sigma$-algebra generated by the random variable $W\left(\tau_{2}\right)-W\left(\tau_{1}\right)$ for all $\tau_{1}, \tau_{2}$ such that $s \leq \tau_{1} \leq \tau_{2} \leq t$ and $\mathcal{N}$ is the collection of $\mathbb{P}$-null sets of $\overline{\mathscr{F}}$.

Note that

$$
\vartheta_{\tau}^{-1} \mathscr{F}_{s}^{t}=\mathscr{F}_{s+\tau}^{t+\tau}
$$

so $\left(\Omega, \mathscr{F}, \mathbb{P},\left(\vartheta_{t}\right)_{t \in \mathbb{R}},\left(\mathscr{F}_{s}^{t}\right)_{s \leq t}\right)$ is a filtered metric dynamical system (see [32, pages 72 and 91] for more details). In addition, it is important to note that the measurability of $\vartheta$ is not true if we replace $\mathscr{F}$ by its completion; see [32, page 547] for details. 
In this paper, the solution of problem (10)-(11) is interpreted in a mild sense:

$$
\begin{gathered}
u(t)=S(t) u^{0}(0)+\int_{0}^{t} S(t-s)\left(f\left(u^{s}\right)+g\right) d s \\
+\int_{0}^{t} S(t-s) d W(s), \quad t>0, \\
u(t)=u^{0}(t), \quad t \in[-v, 0],
\end{gathered}
$$

$\mathbb{P}$ a.s. for any $u^{0} \in \mathscr{C}$, where $S(t)$ is the analytic semigroup on $L^{2}\left(\mathbb{R}^{d}\right)$ generated by $\Delta-\lambda I$. By the theory in [35], we deal with $(22)$ on the complete probability space $(\Omega, \overline{\mathscr{F}}, \mathbb{P})$.

We now associate a continuous random dynamical system with the stochastic retarded reaction-diffusion equation (10)-(11) over $\left(\Omega, \mathscr{F}, \mathbb{P},\left(\vartheta_{t}\right)_{t \in \mathbb{R}}\right)$. To this end, we introduce an auxiliary Ornstein-Uhlenbeck process on $\left(\Omega, \mathscr{F}, \mathbb{P},\left(\vartheta_{t}\right)_{t \in \mathbb{R}}\right)$ and transform the stochastic retarded reaction-diffusion equation into a random one. Let

$z(t, \omega)$

$$
= \begin{cases}\int_{-\infty}^{t}(\Delta-\lambda I) S(t-s)(W(s, \omega)-W(t, \omega)) d s, & \omega \in \widetilde{\Omega}, \\ 0, & \omega \notin \widetilde{\Omega} .\end{cases}
$$

Then by (18), (23) is well defined. The process $z(t), t \in \mathbb{R}$, is a stationary, Gaussian process. By Lemma 5.13 in [35], we can see that it is a mild solution of the linear equation

$$
d z(t)=(\Delta-\lambda I) z(t) d t+d W(t)
$$

That is, for all $t \in \mathbb{R}$ and $\mathbb{P}$ a.s.

$$
z(t)=\int_{-\infty}^{t} S(t-s) d W(s) .
$$

Moreover, the random variable $\|z(0, \omega)\|$ is tempered, and, for each $\omega \in \Omega$, the mapping $t \rightarrow z(t, \omega)$ is continuous.

Setting $v(t)=u(t)-z(t)$ for $t \geq-v$ in (22), then by (25), we obtain a deterministic equation, $\mathbb{P}$ a.s. in $\Omega$,

$$
\begin{gathered}
v(t)=S(t) v^{0}(0)+\int_{0}^{t} S(t-s)\left(f\left(v^{s}+z^{s}\right)+g\right) d s, \quad t>0, \\
v(t)=v^{0}(t), \quad t \in[-v, 0],
\end{gathered}
$$

which is the mild form of the evolution equation

$$
\frac{d v}{d t}=\Delta v-\lambda v+f\left(v^{t}+z^{t}\right)+g
$$

with the initial condition

$$
v(t)=v^{0}(t), \quad t \in[-v, 0] .
$$

Here $v^{0}(t)=u^{0}(t)-z^{0}(t, \omega), t \in[-v, 0]$.

Problem (27)-(28) is a deterministic partial functional differential equation with random coefficients, which can be solved pathwise. We now establish the following result for problem (27)-(28).
Theorem 11. Let $T>0$ and $\omega \in \Omega$ be fixed. Then the following properties hold:

(1) for each $v^{0} \in \mathscr{C}$, problem (27)-(28) has a unique mild solution $v\left(\cdot, \omega, v^{0}\right)$ that belongs to

$$
\begin{gathered}
C\left([-v, T] ; L^{2}\left(\mathbb{R}^{d}\right)\right) \cap W^{1,2}\left(\varepsilon, T ; L^{2}\left(\mathbb{R}^{d}\right)\right) \\
\cap L^{2}\left(\varepsilon, T ; H^{2}\left(\mathbb{R}^{d}\right)\right),
\end{gathered}
$$

for any $\varepsilon \in(0, T)$, and for a.e. $t \in[0, T]$

$$
\frac{d v}{d t}=\Delta v-\lambda v+f\left(v^{t}+z^{t}\right)+g
$$

(2) Let $v_{1}\left(\cdot, \omega, v_{1}^{0}\right)$ and $v_{2}\left(\cdot, \omega, v_{2}^{0}\right)$ be the mild solutions of problem (27)-(28) for the initial data $v_{1}^{0}$ and $v_{2}^{0}$, respectively. Then there exists a constant $c(T)>0$ such that for all $t \in[0, T]$

$$
\left\|v_{1}^{t}\left(\cdot, \omega, v_{1}^{0}\right)-v_{2}^{t}\left(\cdot, \omega, v_{2}^{0}\right)\right\|_{\mathscr{C}} \leq\left\|v_{1}^{0}-v_{2}^{0}\right\|_{\mathscr{C}} e^{(c(T)-\lambda) t+\lambda \nu}
$$

Proof. (1) By $\left(\mathrm{A}_{1}\right)-\left(\mathrm{A}_{2}\right)$, following the same lines of Theorem 6.1.4 in [36], one can show that, for each $v^{0} \in \mathscr{C}$, there exists a $T_{\max } \leq \infty$ such that (26) has a unique solution $v$ on $\left[0, T_{\max }\right)$. Moreover, if $T_{\max }<\infty$, then

$$
\underset{t \uparrow T_{\max }}{\lim \sup }\left\|v^{t}\right\|_{\mathscr{C}}=\infty .
$$

We prove now that this local solution is a global one. For fixed $T \in\left(0, T_{\max }\right)$, by regularity of mild solutions for an analytic semigroup [37, page 145], we inform that

$$
\begin{gathered}
v \in C\left([-v, T] ; L^{2}\left(\mathbb{R}^{d}\right)\right) \cap W^{1,2}\left(\varepsilon, T ; L^{2}\left(\mathbb{R}^{d}\right)\right) \\
\cap L^{2}\left(\varepsilon, T ; H^{2}\left(\mathbb{R}^{d}\right)\right),
\end{gathered}
$$

for any $\varepsilon \in(0, T)$, and (27) holds for a.e. $t \in[0, T]$. Then, taking the inner product of (27) with $v$ in $L^{2}\left(\mathbb{R}^{d}\right)$, we get that

$$
\frac{1}{2} \frac{d}{d t}\|v\|^{2}+\lambda\|v\|^{2}+\|\nabla v\|^{2}=\left(f\left(v^{t}+z^{t}\right), v\right)+(g, v) .
$$

By $\left(\mathrm{A}_{4}\right)$, we can choose $\beta>0$ small enough such that $2 \lambda>$ $2 c_{f}+\beta$. Using the Young inequality, we find that

$$
\begin{aligned}
& \left(f\left(v^{t}+z^{t}\right), v\right) \\
& \leq\left\|f\left(v^{t}+z^{t}\right)\right\|\|v\| \leq \frac{c_{f}}{2}\|v\|^{2}+\frac{1}{2 c_{f}}\left\|f\left(v^{t}+z^{t}\right)\right\|^{2}, \\
& (g, v) \leq\|g\|\|v\| \leq \frac{\beta}{2}\|v\|^{2}+\frac{1}{2 \beta}\|g\|^{2} .
\end{aligned}
$$

Then it follows from (34) and (35) that

$$
\begin{aligned}
\frac{d}{d t}\|v\|^{2}+2\|\nabla v\|^{2} \leq & -\left(2 \lambda-c_{f}-\beta\right)\|v\|^{2} \\
& +\frac{1}{c_{f}}\left\|f\left(v^{t}+z^{t}\right)\right\|^{2}+\frac{1}{\beta}\|g\|^{2} .
\end{aligned}
$$


Choose $\alpha \in\left(0, \alpha_{0}\right)$ small enough such that $2 \lambda>2 c_{f}+\alpha+\beta$. Then by (36), we obtain

$$
\begin{aligned}
\frac{d}{d t}\left(e^{\alpha t}\|v\|^{2}\right)+2 e^{\alpha t}\|\nabla v\|^{2} \\
\leq-\left(2 \lambda-c_{f}-\alpha-\beta\right) e^{\alpha t}\|v\|^{2} \\
+\frac{e^{\alpha t}}{c_{f}}\left\|f\left(v^{t}+z^{t}\right)\right\|^{2}+\frac{e^{\alpha t}}{\beta}\|g\|^{2} .
\end{aligned}
$$

Now, we can also choose $\gamma>0$ such that $2 \lambda>(2+\gamma) c_{f}+\alpha+\beta$. Integrating (37) over $[0, t](t \in[0, T])$ leads to

$$
\begin{aligned}
e^{\alpha t}\|v(t)\|^{2} \leq & \|v(0)\|^{2}-\left(2 \lambda-c_{f}-\alpha-\beta\right) \int_{0}^{t} e^{\alpha s}\|v(s)\|^{2} d s \\
& +\frac{1}{c_{f}} \int_{0}^{t} e^{\alpha s}\left\|f\left(v^{s}+z^{s}\right)\right\|^{2} d s+\frac{\|g\|^{2}}{\beta} \int_{0}^{t} e^{\alpha s} d s .
\end{aligned}
$$

Using the Young inequality and $\left(\mathrm{A}_{3}\right)$, we find that

$$
\begin{gathered}
\frac{1}{c_{f}} \int_{0}^{t} e^{\alpha s}\left\|f\left(v^{s}+z^{s}\right)\right\|^{2} d s \\
\leq c_{f} \int_{-v}^{t} e^{\alpha s}\|v(s)+z(s)\|^{2} d s \\
\leq c_{f} \int_{0}^{t} e^{\alpha s}\left[(1+\gamma)\|v(s)\|^{2}\right. \\
\left.\quad+\left(1+\gamma^{-1}\right)\|z(s)\|^{2}\right] d s \\
+c_{f} \int_{-v}^{0} e^{\alpha s}\|v(s)+z(s)\|^{2} d s .
\end{gathered}
$$

Then by (38) and (39), we obtain

$$
\begin{aligned}
e^{\alpha t}\|v(t)\|^{2} \leq & -\left(2 \lambda-(2+\gamma) c_{f}-\alpha-\beta\right) \\
& \times \int_{0}^{t} e^{\alpha s}\|v(s)\|^{2} d s \\
& +\|v(0)\|^{2}+\frac{\|g\|^{2}}{\alpha \beta} e^{\alpha t} \\
& +c_{f} \int_{-v}^{0} e^{\alpha s}\|v(s)+z(s)\|^{2} d s \\
& +c_{1} \int_{0}^{t} e^{\alpha s}\|z(s)\|^{2} d s \\
\leq & \|v(0)\|^{2}+\frac{\|g\|^{2}}{\alpha \beta} e^{\alpha t} \\
& +2 v c_{f}\left[\left\|v^{0}\right\|_{\mathscr{C}}^{2}+\left\|z^{0}\right\|_{\mathscr{C}}^{2}\right] \\
& +c_{1} \int_{0}^{t} e^{\alpha s}\|z(s)\|^{2} d s,
\end{aligned}
$$

where $c_{1}=c_{f}\left(1+\gamma^{-1}\right)$. Consequently,

$$
\begin{aligned}
\|v(t)\|^{2} \leq & {\left[\left(1+2 v c_{f}\right)\left\|v^{0}\right\|_{\mathscr{C}}^{2}+2 v c_{f}\left\|z^{0}\right\|_{\mathscr{C}}^{2}\right] e^{-\alpha t} } \\
& +c_{1} \int_{0}^{t} e^{\alpha(s-t)}\|z(s)\|^{2} d s+\frac{\|g\|^{2}}{\alpha \beta} .
\end{aligned}
$$

Hence, for fixed $\sigma \in[-\nu, 0]$, we get that, for $t \in(-\sigma, T]$,

$$
\begin{aligned}
\|v(t+\sigma)\|^{2} \leq & {\left[\left(1+2 \nu c_{f}\right)\left\|v^{0}\right\|_{\mathscr{C}}^{2}+2 \nu c_{f}\left\|z^{0}\right\|_{\mathscr{C}}^{2}\right] e^{-\alpha(t+\sigma)} } \\
& +c_{1} \int_{0}^{t+\sigma} e^{\alpha(s-t-\sigma)}\|z(s)\|^{2} d s+\frac{\|g\|^{2}}{\alpha \beta} \\
\leq & {\left[\left(1+2 v c_{f}\right)\left\|v^{0}\right\|_{\mathscr{C}}^{2}+2 \nu c_{f}\left\|z^{0}\right\|_{\mathscr{C}}^{2}\right] e^{\alpha(\nu-t)} } \\
& +c_{1} e^{\alpha \nu} \int_{0}^{t} e^{\alpha(s-t)}\|z(s)\|^{2} d s+\frac{\|g\|^{2}}{\alpha \beta},
\end{aligned}
$$

and, for $t \in[0,-\sigma]$,

$$
\begin{aligned}
\|v(t+\sigma)\|^{2} & \leq\left\|v^{0}\right\|_{\mathscr{C}}^{2} \\
& \leq\left[\left(1+2 v c_{f}\right)\left\|v^{0}\right\|_{\mathscr{C}}^{2}+2 v c_{f}\left\|z^{0}\right\|_{\mathscr{C}}^{2}\right] e^{\alpha(\nu-t)} .
\end{aligned}
$$

In view of (42) and (43), we find that, for all $t \in[0, T]$,

$$
\begin{aligned}
\left\|v^{t}\right\|_{\mathscr{C}}^{2} \leq & {\left[\left(1+2 v c_{f}\right)\left\|v^{0}\right\|_{\mathscr{C}}^{2}+2 v c_{f}\left\|z^{0}\right\|_{\mathscr{C}}^{2}\right] e^{\alpha(v-t)} } \\
& +c_{1} e^{\alpha v} \int_{0}^{t} e^{\alpha(s-t)}\|z(s)\|^{2} d s+\frac{\|g\|^{2}}{\alpha \beta} .
\end{aligned}
$$

Therefore, for all $t \in[0, T]$,

$$
\begin{aligned}
\left\|v^{t}\right\|_{\mathscr{C}}^{2} \leq & {\left[\left(1+2 v c_{f}\right)\left\|v^{0}\right\|_{\mathscr{C}}^{2}+2 v c_{f}\left\|z^{0}\right\|_{\mathscr{C}}^{2}\right] e^{\alpha \nu} } \\
& +c_{1} e^{\alpha \nu} \int_{0}^{T}\|z(s)\|^{2} d s+\frac{\|g\|^{2}}{\alpha \beta}
\end{aligned}
$$

which, together with (32), implies that $T_{\max }=\infty$. This proves property (1).

(2) By (44), there exists a constant $r(T)>0$ such that

$$
\left\|v_{1}^{t}\right\|_{\mathscr{C}} \leq r(T), \quad\left\|v_{2}^{t}\right\|_{\mathscr{C}} \leq r(T)
$$

Then from $\left(\mathrm{A}_{2}\right)$ and (26), we have that for $t \in[0, T]$

$$
\begin{aligned}
\left\|v_{1}(t)-v_{2}(t)\right\| \leq & e^{-\lambda t}\left\|v_{1}^{0}(0)-v_{2}^{0}(0)\right\| \\
& +l_{f}(r(T)) \int_{0}^{t} e^{-\lambda(t-s)}\left\|v_{1}^{s}-v_{2}^{s}\right\|_{\mathscr{C}} d s .
\end{aligned}
$$


Hence, for fixed $\sigma \in[-\nu, 0]$, we get that, for $t \in(-\sigma, T]$,

$$
\begin{aligned}
& \left\|v_{1}(t+\sigma)-v_{2}(t+\sigma)\right\| \\
& \leq e^{-\lambda(t+\sigma)}\left\|v_{1}^{0}(0)-v_{2}^{0}(0)\right\| \\
& \quad+l_{f}(r(T)) \int_{0}^{t+\sigma} e^{-\lambda(t+\sigma-s)}\left\|v_{1}^{s}-v_{2}^{s}\right\|_{\mathscr{C}} d s \\
& \leq e^{\lambda(v-t)}\left\|v_{1}^{0}-v_{2}^{0}\right\|_{\mathscr{C}} \\
& \quad+l_{f}(r(T)) e^{\lambda \nu} \int_{0}^{t} e^{-\lambda(t-s)}\left\|v_{1}^{s}-v_{2}^{s}\right\|_{\mathscr{C}} d s,
\end{aligned}
$$

and, for $t \in[0,-\sigma]$,

$$
\left\|v_{1}(t+\sigma)-v_{2}(t+\sigma)\right\| \leq\left\|v_{1}^{0}-v_{2}^{0}\right\|_{\mathscr{C}} \leq e^{\lambda(\nu-t)}\left\|v_{1}^{0}-v_{2}^{0}\right\|_{\mathscr{C}} .
$$

In view of (48) and (49), we find that, for all $t \in[0, T]$,

$$
\begin{aligned}
\left\|v_{1}^{t}-v_{2}^{t}\right\| \leq & e^{\lambda(\nu-t)}\left\|v_{1}^{0}-v_{2}^{0}\right\|_{\mathscr{C}} \\
& +l_{f}(r(T)) e^{\lambda v} \int_{0}^{t} e^{-\lambda(t-s)}\left\|v_{1}^{s}-v_{2}^{s}\right\|_{\mathscr{C}} d s .
\end{aligned}
$$

The Gronwall inequality implies that, for all $t \in[0, T]$,

$$
\left\|v_{1}^{t}-v_{2}^{t}\right\|_{\mathscr{C}} \leq\left\|v_{1}^{0}-v_{2}^{0}\right\|_{\mathscr{C}} e^{\left(l_{f}(r(T)) e^{\lambda \nu}-\lambda\right) t+\lambda \nu}
$$

This prove property (2). The proof is complete.

Conversely, if, for each $\omega \in \Omega, v\left(t, \omega, v^{0}\right) \quad \epsilon$ $C\left([-v, \infty) ; L^{2}\left(\mathbb{R}^{d}\right)\right)$ is a mild solution of problem (27)-(28) with $v^{0}(\cdot)=u^{0}(\cdot)-z^{0}(\cdot, \omega)$, then by (25) the continuous process

$$
u\left(t, \omega, u^{0}\right)=v\left(t, \omega, v^{0}\right)+z(t, \omega)
$$

is a mild solution of problem (10)-(11).

Theorem 12. Problem (27)-(28) generates a continuous random dynamical system $\phi$ over $\left(\Omega, \mathscr{F}, \mathbb{P},(\vartheta)_{t \in \mathbb{R}}\right)$, where

$$
\phi\left(t, \omega, v^{0}\right)=v^{t}\left(\cdot, \omega, v^{0}\right), \quad \text { for } t \geq 0, \omega \in \Omega, v^{0} \in \mathscr{C} .
$$

Moreover, if one defines $\psi$ by

$$
\psi\left(t, \omega, u^{0}\right)=u^{t}\left(\cdot, \omega, u^{0}\right), \quad \text { for } t \geq 0, \omega \in \Omega, u^{0} \in \mathscr{C},
$$

then $\psi$ is another continuous random dynamical system associated with problem (10)-(11).

Proof. By a classical successive approximation argument, one can easily show that, for fixed $v^{0} \in \mathscr{C}, v\left(t, \omega, v^{0}\right)$ is an $\mathscr{F}_{t}$-adapted continuous process. Hence, for fixed $v^{0} \in \mathscr{C}$, $\phi\left(t, \omega, v^{0}\right)$ is also an $\mathscr{F}_{t}$-adapted continuous process. On the other hand, from property (2) of Theorem 11, it follows that, for fixed $t \geq 0$ and $\omega \in \Omega, \phi(t, \omega, \cdot):[0, \infty) \times \mathscr{C} \rightarrow \mathscr{C}$ is continuous. Consequently, $\phi\left(t, \omega, v^{0}\right)$ is $\left(\mathscr{B}\left(\mathbb{R}^{+}\right) \otimes \mathscr{F} \otimes\right.$ $\mathscr{B}(\mathscr{C}), \mathscr{B}(\mathscr{C})$ )-measurable.

By (26) we have that, for $s, t \geq 0$ and $\sigma \in[-\nu, 0]$,

$$
\begin{aligned}
& \phi\left(t, \vartheta_{s} \omega, \phi\left(s, \omega, v^{0}\right)\right)(\sigma) \\
& =S(t+\sigma) \phi\left(s, \omega, v^{0}\right)(0) \\
& \quad+\int_{0}^{t+\sigma} S(t+\sigma-\tau) \\
& \quad \times\left(f\left(\phi\left(\tau, \vartheta_{s} \omega, \phi\left(s, \omega, v^{0}\right)\right)+z^{\tau}\left(\vartheta_{s} \omega\right)\right)+g\right) d \tau .
\end{aligned}
$$

Then again by (26) we get

$$
\begin{aligned}
& \phi\left(t, \vartheta_{s} \omega, \phi\left(s, \omega, v^{0}\right)\right)(\sigma) \\
& =S(t+s+\sigma) v^{0}(0) \\
& \quad+\int_{0}^{s} S(s-\tau) \\
& \quad \times\left(f\left(\phi\left(\tau, \omega, v^{0}\right)+z^{\tau}(\omega)\right)+g\right) d \tau \\
& +\int_{s}^{t+s+\sigma} \quad S(t+s+\sigma-\tau) \\
& \quad \times\left(f\left(\phi\left(\tau-s, \vartheta_{s} \omega, \phi\left(s, \omega, v^{0}\right)\right)+z^{\tau}(\omega)\right)+g\right) d \tau .
\end{aligned}
$$

For each $\omega \in \Omega$ consider

$$
\Phi\left(\tau, \omega, v^{0}\right)= \begin{cases}\phi\left(\tau, \omega, v^{0}\right), & \text { if } 0 \leq \tau \leq s \\ \phi\left(\tau-s, \vartheta_{s} \omega, \phi\left(s, \omega, v^{0}\right)\right), & \text { if } s<\tau \leq t+s\end{cases}
$$

Then for $\tau=t+s$ we have

$$
\Phi\left(t+s, \omega, v^{0}\right)=\phi\left(t, \vartheta_{s} \omega, \phi\left(s, \omega, v^{0}\right)\right) \quad \text { for } s, t \geq 0 .
$$

It follows from (56) that

$$
\begin{aligned}
& \Phi\left(t+s, \omega, v^{0}\right)(\sigma) \\
& =S(t+s+\sigma) v^{0}(0) \\
& \quad+\int_{0}^{t+s+\sigma} S(t+s+\sigma-\tau) \\
& \quad \times\left(f\left(\Phi\left(\tau, \omega, v^{0}\right)+z^{\tau}(\omega)\right)+g\right) d \tau
\end{aligned}
$$

for all $\sigma \in[-v, 0]$. By the uniqueness of the solution of (26) we find that

$$
\Phi\left(t+s, \omega, v^{0}\right)=\phi\left(t+s, \omega, v^{0}\right)
$$

while (58) implies

$$
\phi\left(t+s, \omega, v^{0}\right)=\phi\left(t, \vartheta_{s} \omega, \phi\left(s, \omega, v^{0}\right)\right) \quad \text { for } s, t \geq 0 .
$$

Therefore, $\phi$ is a continuous random dynamical system. 
As for $\psi$, noticing that

$$
\psi\left(t, \omega, u^{0}\right)=\phi\left(t, \omega, u^{0}-z^{0}(\omega)\right)+z^{t}(\omega)
$$$$
\text { for } t \geq 0, \quad \omega \in \Omega, \quad u^{0} \in \mathscr{C} \text {, }
$$

we get from (61) that, for $s, t \geq 0$,

$$
\begin{aligned}
\psi & \left(t, \vartheta_{s} \omega, \psi\left(s, \omega, u^{0}\right)\right) \\
& =\phi\left(t, \vartheta_{s} \omega, \phi\left(s, \omega, u^{0}-z^{0}(\omega)\right)\right)+z^{t}\left(\vartheta_{s} \omega\right) \\
& =\phi\left(t+s, \omega, u^{0}-z^{0}(\omega)\right)+z^{t+s}(\omega) \\
& =\psi\left(t+s, \omega, u^{0}\right) .
\end{aligned}
$$

Therefore, $\psi$ is also a continuous random dynamical system. Furthermore, $\phi$ and $\psi$ are conjugated random dynamical systems; that is,

$$
\psi(t, \omega, T(\omega, \xi))=T\left(\vartheta_{t} \omega, \phi(t, \omega, \xi)\right), \quad \text { for any } \xi \in \mathscr{C} \text {, }
$$

where, for every $\omega \in \Omega, T(\omega, \xi)=\xi+z^{0}(\omega)$ is a homeomorphism of $\mathscr{C}$. The proof is complete.

\section{Existence of Random Attractors}

In this section, we prove the existence of a $\mathscr{D}$-random attractor for the random dynamical system $\psi$ associated with the stochastic retarded reaction-diffusion equation (10)(11) on $\mathbb{R}^{d}$. We first establish the existence of a $\mathscr{D}$-random attractor for its conjugated random dynamical system $\phi$, then the existence of a $\mathscr{D}$-random attractor for $\psi$ follows from the conjugation relation between $\phi$ and $\psi$. To this end, we will derive uniform estimates on the mild solutions of problem (27)-(28) when $t \rightarrow \infty$ with the purpose of proving the existence of a bounded random absorbing set and the asymptotic compactness for $\phi$. In particular, we will show that the tails of the solutions, that is, solutions evaluated at large value of $|x|$, are uniformly small when time is sufficiently large.

From now on, we always assume that $\mathscr{D}$ is the collection of all tempered subsets of $\mathscr{C}$ with respect to $\left(\Omega, \mathscr{F}, \mathbb{P},\left(\vartheta_{t}\right)_{t \in \mathbb{R}}\right)$. The next lemma shows that $\phi$ has a random absorbing set in D.

Lemma 13. There exists $K \in \mathscr{D}$ such that $K$ is a random absorbing set for $\phi$ in $\mathscr{D}$; that is, for any $B \in \mathscr{D}$ and $\mathbb{P}$ a.e. $\omega \in \Omega$, there exists $T_{B}(\omega)>0$ such that

$$
\phi\left(t, \vartheta_{-t} \omega, B\left(\vartheta_{-t} \omega\right)\right) \subseteq K(\omega) \quad \forall t \geq T_{B}(\omega)
$$

Proof. Replacing $\omega$ with $\vartheta_{-t} \omega$ in (41) and (44), we get that, for all $t \geq 0$,

$$
\begin{aligned}
\| v( & \left.t, \vartheta_{-t} \omega, v^{0}\left(\vartheta_{-t} \omega\right)\right) \|^{2} \\
\leq & {\left[\left(1+2 \nu c_{f}\right)\left\|v^{0}\left(\vartheta_{-t} \omega\right)\right\|_{\mathscr{C}}^{2}+2 \nu c_{f}\left\|z^{0}\left(\vartheta_{-t} \omega\right)\right\|_{\mathscr{C}}^{2}\right] e^{-\alpha t} } \\
& +c_{1} \int_{0}^{t} e^{\alpha(s-t)}\left\|z\left(s, \vartheta_{-t} \omega\right)\right\|^{2} d s+\frac{\|g\|^{2}}{\alpha \beta} \\
\leq & {\left[\left(1+2 \nu c_{f}\right)\left\|v^{0}\left(\vartheta_{-t} \omega\right)\right\|_{\mathscr{C}}^{2}+2 \nu c_{f}\left\|z^{0}\left(\vartheta_{-t} \omega\right)\right\|_{\mathscr{C}}^{2}\right] e^{-\alpha t} } \\
& +c_{1} \int_{-\infty}^{0} e^{\alpha s}\left\|z\left(0, \vartheta_{s} \omega\right)\right\|^{2} d s+\frac{\|g\|^{2}}{\alpha \beta}, \\
\left\|v^{t}\left(\vartheta_{-t} \omega, v^{0}\left(\vartheta_{-t} \omega\right)\right)\right\|_{\mathscr{C}}^{2} & {\left[\left(1+2 \nu c_{f}\right)\left\|v^{0}\left(\vartheta_{-t} \omega\right)\right\|_{\mathscr{C}}^{2}+2 \nu c_{f}\left\|z^{0}\left(\vartheta_{-t} \omega\right)\right\|_{\mathscr{C}}^{2}\right] e^{\alpha(\nu-t)} } \\
\leq & +c_{1} e^{\alpha \nu} \int_{0}^{t} e^{\alpha(s-t)}\left\|z\left(s, \vartheta_{-t} \omega\right)\right\|^{2} d s+\frac{\|g\|^{2}}{\alpha \beta} \\
\leq & {\left[\left(1+2 v c_{f}\right)\left\|v^{0}\left(\vartheta_{-t} \omega\right)\right\|_{\mathscr{C}}^{2}+2 \nu c_{f}\left\|z^{0}\left(\vartheta_{-t} \omega\right)\right\|_{\mathscr{C}}^{2}\right] e^{\alpha(\nu-t)} e^{\alpha s}\left\|z\left(0, \vartheta_{s} \omega\right)\right\|^{2} d s+\frac{\|g\|^{2}}{\alpha \beta} . } \\
& {[(66)}
\end{aligned}
$$

By assumption, $B \in \mathscr{D}$ is tempered. On the other hand, by Remark 6, $\left\|z^{0}(\omega)\right\|_{\mathscr{C}}^{2}$ is also tempered. Therefore, if $v^{0}\left(\vartheta_{-t} \omega\right) \epsilon$ $B\left(\vartheta_{-t} \omega\right)$, then there exists $T_{B}(\omega)>0$ such that, for all $t \geq$ $T_{B}(\omega)$,

$$
\begin{aligned}
& {\left[\left(1+2 \nu c_{f}\right)\left\|v^{0}\left(\vartheta_{-t} \omega\right)\right\|_{\mathscr{C}}^{2}+2 \nu c_{f}\left\|z^{0}\left(\vartheta_{-t} \omega\right)\right\|_{\mathscr{C}}^{2}\right] e^{-\alpha t}} \\
& \leq 1+r(\omega)
\end{aligned}
$$

where

$$
r(\omega)=\int_{-\infty}^{0} e^{\alpha s}\left\|z\left(0, \vartheta_{s} \omega\right)\right\|^{2} d s
$$

is tempered by Remark 6 . Then it follows from (66), (67), and (68) that for all $t \geq T_{B}(\omega)$,

$$
\begin{gathered}
\left\|v\left(t, \vartheta_{-t} \omega, v^{0}\left(\vartheta_{-t} \omega\right)\right)\right\|^{2} \leq\left(c_{1}+1\right) r(\omega)+\frac{\|g\|^{2}}{\alpha \beta}+1, \quad(70) \\
\left\|v^{t}\left(\vartheta_{-t} \omega, v^{0}\left(\vartheta_{-t} \omega\right)\right)\right\|_{\mathscr{C}}^{2} \leq\left(c_{1}+1\right) e^{\alpha \nu} r(\omega)+\frac{\|g\|^{2}}{\alpha \beta}+1 .
\end{gathered}
$$

Given $\omega \in \Omega$, we define

$$
K(\omega)=\left\{\xi \in \mathscr{C}:\|\xi\|_{\mathscr{C}}^{2} \leq r_{1}(\omega)\right\},
$$


where

$$
r_{1}(\omega)=\left(c_{1}+1\right) e^{\alpha v} r(\omega)+\frac{\|g\|^{2}}{\alpha \beta}+1
$$

is tempered. Then $K \in \mathscr{D}$. Further, (71) indicates that $K$ is a random absorbing set for $\phi$ in $\mathscr{D}$, which completes the proof.

We next derive uniform estimates for $v$ in $H^{1}\left(\mathbb{R}^{d}\right)$. From property (1) of Theorem 11 and the fact that [37, page 165]

$$
\begin{gathered}
W^{1,2}\left(\varepsilon, T ; L^{2}\left(\mathbb{R}^{d}\right)\right) \cap L^{2}\left(\varepsilon, T ; H^{2}\left(\mathbb{R}^{d}\right)\right) \\
\quad C C\left([\varepsilon, T] ; H^{1}\left(\mathbb{R}^{d}\right)\right),
\end{gathered}
$$

for any $0<\varepsilon<T<\infty$, we get that $v \in C\left((0, \infty) ; H^{1}\left(\mathbb{R}^{d}\right)\right)$.

Lemma 14. Let $B \in \mathscr{D}$ and $v^{0}(\omega) \in B(\omega)$. Then for $\mathbb{P}$ a.e. $\omega \in \Omega$, the solution $v\left(t, \omega, v^{0}(\omega)\right)$ of problem (27)-(28) satisfies, for all $t \geq T_{B}(\omega)$,

$$
\begin{gathered}
\int_{t}^{t+1}\left\|v^{s}\left(\vartheta_{-t-1} \omega, v^{0}\left(\vartheta_{-t-1} \omega\right)\right)\right\|_{\mathscr{C}}^{2} d s \leq r_{2}(\omega), \\
\int_{t}^{t+1}\left\|\nabla v\left(s, \vartheta_{-t-1} \omega\right)\right\|^{2} d s \leq r_{3}(\omega),
\end{gathered}
$$

where $r_{2}$ and $r_{3}$ are tempered and $T_{B}$ is the random function in Lemma 13.

Proof. Replacing $\omega$ with $\vartheta_{-\tau-1} \omega$ in (44), we get that, for all $\tau \geq 0$ and $t \in[\tau, \tau+1]$,

$$
\begin{aligned}
\left\|v^{t}\left(\vartheta_{-\tau-1} \omega, v^{0}\left(\vartheta_{-\tau-1} \omega\right)\right)\right\|_{\mathscr{C}}^{2} & \\
\leq & {\left[\left(1+2 v c_{f}\right)\left\|v^{0}\left(\vartheta_{-\tau-1} \omega\right)\right\|_{\mathscr{C}}^{2}\right.} \\
& \left.+2 v c_{f}\left\|z^{0}\left(\vartheta_{-\tau-1} \omega\right)\right\|_{\mathscr{C}}^{2}\right] e^{\alpha(\nu-t)} \\
& +c_{1} e^{\alpha \nu} \int_{0}^{t} e^{\alpha(s-t)}\left\|z\left(s, \vartheta_{-\tau-1} \omega\right)\right\|^{2} d s+\frac{\|g\|^{2}}{\alpha \beta} .
\end{aligned}
$$

Integrating (76) over the interval $[\tau, \tau+1]$ leads to

$$
\begin{aligned}
\int_{\tau}^{\tau+1} & \left\|v^{t}\left(\vartheta_{-\tau-1} \omega, v^{0}\left(\vartheta_{-\tau-1} \omega\right)\right)\right\|_{\mathscr{C}}^{2} d t \\
\leq & {\left[\left(1+2 \nu c_{f}\right)\left\|v^{0}\left(\vartheta_{-\tau-1} \omega\right)\right\|_{\mathscr{C}}^{2}+2 v c_{f}\left\|z^{0}\left(\vartheta_{-\tau-1} \omega\right)\right\|_{\mathscr{C}}^{2}\right] } \\
& \times \int_{\tau}^{\tau+1} e^{\alpha(\nu-t)} d t \\
& +c_{1} e^{\alpha \nu} \int_{\tau}^{\tau+1} \int_{0}^{t} e^{\alpha(s-t)}\left\|z\left(0, \vartheta_{s-\tau-1} \omega\right)\right\|^{2} d s d t+\frac{\|g\|^{2}}{\alpha \beta}
\end{aligned}
$$

$$
\begin{aligned}
\leq & {\left[\left(1+2 v c_{f}\right)\left\|v^{0}\left(\vartheta_{-\tau-1} \omega\right)\right\|_{\mathscr{C}}^{2}\right.} \\
& \left.+2 v c_{f}\left\|z^{0}\left(\vartheta_{-\tau-1} \omega\right)\right\|_{\mathscr{C}}^{2}\right] e^{\alpha(\gamma-\tau)} \\
& +c_{1} e^{\alpha(v+1)} r(\omega)+\frac{\|g\|^{2}}{\alpha \beta},
\end{aligned}
$$

which, together with (68), implies that, for all $t \geq T_{B}(\omega)$,

$$
\begin{aligned}
\int_{t}^{t+1} & \left\|v^{s}\left(\vartheta_{-t-1} \omega, v^{0}\left(\vartheta_{-t-1} \omega\right)\right)\right\|_{\mathscr{C}}^{2} d s \\
\leq & {\left[c_{1} e^{\alpha(\nu+1)}+e^{\alpha \nu}\right] r(\omega)+\frac{\|g\|^{2}}{\alpha \beta}+e^{\alpha \nu}:=r_{2}(\omega) . }
\end{aligned}
$$

Obviously, $r_{2}$ is tempered. Integrating (37) over the interval $[t, t+1]$ leads to

$$
\begin{aligned}
e^{\alpha(t+1)}\|v(t+1)\|^{2}-e^{\alpha t}\|v(t)\|^{2} \\
+2 \int_{t}^{t+1} e^{\alpha s}\|\nabla v(s)\|^{2} d s \\
\leq-\left(2 \lambda-c_{f}-\alpha-\beta\right) \int_{t}^{t+1} e^{\alpha s}\|v(s)\|^{2} d s \\
+\frac{\|g\|^{2}}{\beta} \int_{t}^{t+1} e^{\alpha s} d s \\
+\frac{1}{c_{f}} \int_{t}^{t+1} e^{\alpha s}\left\|f\left(v^{s}+z^{s}\right)\right\|^{2} d s .
\end{aligned}
$$

Using the Young inequality and $\left(A_{3}\right)$, we obtain that

$$
\begin{aligned}
& \frac{1}{c_{f}} \int_{t}^{t+1} e^{\alpha s}\left\|f\left(v^{s}+z^{s}\right)\right\|^{2} d s \\
& \leq c_{f} \int_{t-\gamma}^{t+1} e^{\alpha s}\|v(s)+z(s)\|^{2} d s \\
& \leq c_{f} \int_{t}^{t+1} e^{\alpha s}\left[(1+\gamma)\|v(s)\|^{2}+\left(1+\gamma^{-1}\right)\|z(s)\|^{2}\right] d s \\
& \quad+c_{f} \int_{t-\nu}^{t} e^{\alpha s}\|v(s)+z(s)\|^{2} d s .
\end{aligned}
$$

It follows from (79) and (80) that

$$
\begin{aligned}
e^{\alpha(t+1)}\|v(t+1)\|^{2}-e^{\alpha t}\|v(t)\|^{2}+2 \int_{t}^{t+1} e^{\alpha s}\|\nabla v(s)\|^{2} d s \\
\leq- \\
+\frac{\|g\|^{2}}{\beta} e^{\alpha(t+1)}+\int_{t-v}^{t} e^{\alpha s}\|v(s)+z(s)\|^{2} d s \\
+c_{1} \int_{t}^{t+1} e^{\alpha s}\|z(s)\|^{2} d s .
\end{aligned}
$$


Thus,

$$
\begin{aligned}
& 2 \int_{t}^{t+1} e^{\alpha s}\|\nabla v(s)\|^{2} d s \\
& \leq e^{\alpha t}\|v(t)\|^{2}+\int_{t-v}^{t} e^{\alpha s}\|v(s)+z(s)\|^{2} d s \\
& \quad+c_{1} \int_{t}^{t+1} e^{\alpha s}\|z(s)\|^{2} d s+\frac{\|g\|^{2}}{\beta} e^{\alpha(t+1)} .
\end{aligned}
$$

Replacing $\omega$ with $\vartheta_{-\tau-1} \omega$ in (82), we get from (71) that, for $\mathbb{P}$ a.e. $\omega \in \Omega$ and all $t \geq T_{B}(\omega)$,

$$
\begin{aligned}
2 \int_{t}^{t+1} & \left\|\nabla v\left(s, \vartheta_{-t-1} \omega\right)\right\|^{2} d s \\
\leq & \left\|v\left(t, \vartheta_{-t-1} \omega\right)\right\|^{2} \\
& +\int_{t-\nu}^{t}\left\|v\left(s, \vartheta_{-t-1} \omega\right)+z\left(s, \vartheta_{-t-1} \omega\right)\right\|^{2} d s \\
& +c_{1} \int_{t}^{t+1} e^{\alpha(s-t)}\left\|z\left(s, \vartheta_{-t-1} \omega\right)\right\|^{2} d s+\frac{\|g\|^{2}}{\beta} e^{\alpha} \\
\leq & \left\|v\left(t, \vartheta_{-t-1} \omega\right)\right\|^{2} \\
& +2 \int_{t-\nu}^{t}\left[\left\|v\left(s, \vartheta_{-t-1} \omega\right)\right\|^{2}+\left\|z\left(s, \vartheta_{-t-1} \omega\right)\right\|^{2}\right] d s \\
& +c_{1} e^{\alpha} \int_{-1}^{0} e^{\alpha s}\left\|z\left(0, \vartheta_{s} \omega\right)\right\|^{2} d s+\frac{\|g\|^{2}}{\beta} e^{\alpha} \\
\leq & (1+2 \nu)\left\|v^{t}\left(\vartheta_{-t-1} \omega\right)\right\|_{\mathscr{C}}^{2}+2 v\left\|z^{0}\left(\vartheta_{-1} \omega\right)\right\|_{\mathscr{C}}^{2} \\
& +c_{1} e^{\alpha} r(\omega)+\frac{\|g\|^{2}}{\beta} e^{\alpha} . \\
& +c_{1} e^{\alpha} r(\omega)+\frac{\|g\|^{2}}{\beta} e^{\alpha} \\
\leq & +2 \nu) r_{1}\left(\vartheta_{-1} \omega\right)+2 \nu\left\|z^{0}\left(\vartheta_{-1} \omega\right)\right\|_{\mathscr{C}}^{2}
\end{aligned}
$$

Therefore, we have that, for all $t \geq T_{B}(\omega)$,

$$
\begin{aligned}
\int_{t}^{t+1} & \left\|\nabla v\left(s, \vartheta_{-t-1} \omega\right)\right\|^{2} d s \\
\leq & \frac{1+2 v}{2} r_{1}\left(\vartheta_{-1} \omega\right)+v\left\|z^{0}\left(\vartheta_{-1} \omega\right)\right\|_{\mathscr{C}}^{2} \\
& +\frac{c_{1}}{2} e^{\alpha} r(\omega)+\frac{\|g\|^{2}}{2 \beta} e^{\alpha}:=r_{3}(\omega) .
\end{aligned}
$$

By Remark 6, $r_{3}$ is tempered. Then the lemma follows from (78) and (84).
Lemma 15. Let $B \in \mathscr{D}$ and $v^{0}(\omega) \in B(\omega)$. Then for $\mathbb{P}$ a.e. $\omega \in \Omega$, the solution $v\left(t, \omega, v^{0}(\omega)\right)$ of problem (27)-(28) satisfies, for all $t \geq T_{B}(\omega)+\nu+1$ and $\sigma_{1}, \sigma_{2} \in[-v, 0]$,

$$
\begin{gathered}
\left\|\nabla v\left(t, \vartheta_{-t} \omega, v^{0}\left(\vartheta_{-t} \omega\right)\right)\right\|^{2} \leq r_{4}(\omega), \\
\left|\int_{t+\sigma_{1}}^{t+\sigma_{2}}\left\|\Delta v\left(s, \vartheta_{-t} \omega, v^{0}\left(\vartheta_{-t} \omega\right)\right)\right\|^{2} d s\right| \leq r_{5}(\omega),
\end{gathered}
$$

where $r_{4}$ and $r_{5}$ are tempered and $T_{B}$ is the random function in Lemma 13.

Proof. Taking the inner product of (27) with $\Delta v$ in $L^{2}\left(\mathbb{R}^{d}\right)$, we get that

$$
\begin{aligned}
\frac{1}{2} \frac{d}{d t} \| & \nabla v\left\|^{2}+\lambda\right\| \nabla v\left\|^{2}+\right\| \Delta v \|^{2} \\
& =-\left(f\left(v^{t}+z^{t}\right), \Delta v\right)-(g, \Delta v) .
\end{aligned}
$$

Using the Young inequality, we obtain that

$$
\begin{aligned}
&-\left(f\left(v^{t}+z^{t}\right), \Delta v\right) \leq\left\|f\left(v^{t}+z^{t}\right)\right\|\|\Delta v\| \\
& \leq \frac{1}{4}\|\Delta v\|^{2}+\left\|f\left(v^{t}+z^{t}\right)\right\|^{2}, \\
&-(g, \Delta v) \leq\|g\|\|\Delta v\| \leq \frac{1}{4}\|\Delta v\|^{2}+\|g\|^{2} .
\end{aligned}
$$

It follows from (86) and (87) that

$$
\begin{gathered}
\frac{1}{2} \frac{d}{d t}\|\nabla v\|^{2}+\lambda\|\nabla v\|^{2}+\frac{1}{2}\|\Delta v\|^{2} \\
\leq\left\|f\left(v^{t}+z^{t}\right)\right\|^{2}+\|g\|^{2} .
\end{gathered}
$$

Thus,

$$
\frac{d}{d t}\|\nabla v\|^{2}+\|\Delta v\|^{2} \leq 2\left\|f\left(v^{t}+z^{t}\right)\right\|^{2}+2\|g\|^{2} .
$$

Let $T_{B}(\omega)$ be the positive constant in Lemma 13, and take $t \geq$ $T_{B}(\omega)$ and $s \in(t, t+1)$. Integrating (89) over the interval $[s, t+1]$ leads to

$$
\begin{aligned}
& \|\nabla v(t+1)\|^{2} \\
& \leq\|\nabla v(s)\|^{2}+2 \int_{s}^{t+1}\left\|f\left(v^{\tau}+z^{\tau}\right)\right\|^{2} d \tau \\
& \quad+2 \int_{s}^{t+1}\|g\|^{2} d \tau \\
& \leq\|\nabla v(s)\|^{2}+2 \int_{t}^{t+1}\left\|f\left(v^{\tau}+z^{\tau}\right)\right\|^{2} d \tau+2\|g\|^{2} .
\end{aligned}
$$

Integrating the above with respect to $s$ over the interval $[t, t+$ $1]$, we get that

$$
\begin{aligned}
\|\nabla v(t+1)\|^{2} \leq & \int_{t}^{t+1}\|\nabla v(s)\|^{2} d s \\
& +2 \int_{t}^{t+1}\left\|f\left(v^{s}+z^{s}\right)\right\|^{2} d s+2\|g\|^{2} .
\end{aligned}
$$


Replacing $\omega$ with $\vartheta_{-t-1} \omega$ and by $\left(A_{1}\right)-\left(A_{2}\right)$ and Lemmas 13 and 14 , we find that, for all $t \geq T_{B}(\omega)$,

$$
\begin{aligned}
& \left\|\nabla v\left(t+1, \vartheta_{-t-1} \omega, v^{0}\left(\vartheta_{-t-1} \omega\right)\right)\right\|^{2} \\
& \leq \int_{t}^{t+1}\left\|\nabla v\left(s, \vartheta_{-t-1} \omega, v^{0}\left(\vartheta_{-t-1} \omega\right)\right)\right\|^{2} d s+2\|g\|^{2} \\
& \quad+2 \int_{t}^{t+1}\left\|f\left(v^{s}\left(\vartheta_{-t-1} \omega\right)+z^{s}\left(\vartheta_{-t-1} \omega\right)\right)\right\|^{2} d s \\
& \leq \int_{t}^{t+1}\left\|\nabla v\left(s, \vartheta_{-t-1} \omega, v^{0}\left(\vartheta_{-t-1} \omega\right)\right)\right\|^{2} d s+2\|g\|^{2} \\
& \quad+4 l_{f}^{2}\left(p_{1}(\omega)\right) \int_{t}^{t+1}\left[\left\|\left(v^{s} \vartheta_{-t-1} \omega\right)\right\|_{\mathscr{C}}^{2}+\left\|z^{s}\left(\vartheta_{-t-1} \omega\right)\right\|_{\mathscr{C}}^{2}\right] d s \\
& \leq r_{3}(\omega)+4 l_{f}^{2}\left(p_{1}(\omega)\right) \\
& \quad \times\left[r_{2}(\omega)+\sup _{\sigma \in[-1,0]}\left\|z\left(0, \vartheta_{\sigma} \omega\right)\right\|^{2}\right]+2\|g\|^{2}
\end{aligned}
$$

where

$$
p_{1}(\omega)=\sup _{\sigma \in[-1,0]}\left\{\sqrt{r_{1}\left(\vartheta_{\sigma} \omega\right)}+\left\|z\left(0, \vartheta_{\sigma} \omega\right)\right\|\right\}
$$

is tempered by Remark 6 . Then we have that, for all $t \geq$ $T_{B}(\omega)+1$,

$$
\begin{aligned}
& \left\|\nabla v\left(t, \vartheta_{-t} \omega, v^{0}\left(\vartheta_{-t} \omega\right)\right)\right\|^{2} \\
& \leq r_{3}(\omega)+4 l_{f}^{2}\left(p_{1}(\omega)\right) \\
& \quad \times\left[r_{2}(\omega)+\sup _{\sigma \in[-1,0]}\left\|z\left(0, \vartheta_{\sigma} \omega\right)\right\|^{2}\right]+2\|g\|^{2}:=r_{4}(\omega) .
\end{aligned}
$$

By $\left(\mathrm{A}_{2}\right)$, one can easily see that $l_{f}(p)$ is also tempered for any tempered random variable $p$. Hence, $r_{4}$ is tempered.

Let $t \geq v,-v \leq \sigma_{1} \leq \sigma_{2} \leq 0$. Integrating (89) over the interval $\left[t+\sigma_{1}, t+\sigma_{2}\right]$ leads to

$$
\begin{aligned}
& \left\|\nabla v\left(t+\sigma_{2}\right)\right\|^{2}+\int_{t+\sigma_{1}}^{t+\sigma_{2}}\|\Delta v(s)\|^{2} d s \\
& \quad \leq\left\|\nabla v\left(t+\sigma_{1}\right)\right\|^{2}+2 \int_{t+\sigma_{1}}^{t+\sigma_{2}}\left\|f\left(v^{s}+z^{s}\right)\right\|^{2} d s+2\|g\|^{2} d s .
\end{aligned}
$$

Replacing $\omega$ with $\vartheta_{-t} \omega$ in (95) and by $\left(A_{1}\right)-\left(A_{2}\right)$, Lemma 13 , and (94), we find that, for all $t \geq T_{B}(\omega)+\nu+1$ and $\sigma_{1}, \sigma_{2} \in$ $[-v, 0]$,

$$
\begin{aligned}
\int_{t+\sigma_{1}}^{t+\sigma_{2}} & \left\|\Delta v\left(s, \vartheta_{-t} \omega\right)\right\|^{2} d s \\
\leq & \left\|\nabla v\left(t+\sigma_{1}, \vartheta_{-t} \omega\right)\right\|^{2} \\
& +2 \int_{t+\sigma_{1}}^{t+\sigma_{2}}\left\|f\left(v^{s}\left(\vartheta_{-t} \omega\right)+z^{s}\left(\vartheta_{-t} \omega\right)\right)\right\|^{2} d s+2 \nu\|g\|^{2} \\
\leq & 2 v\|g\|^{2}+\sup _{\sigma \in[-\nu, 0]}\left\|\nabla v\left(t+\sigma, \vartheta_{-t} \omega\right)\right\|^{2}+4 l_{f}^{2}\left(p_{2}(\omega)\right) \\
& \times \int_{t-v}^{t}\left[\left\|\left(v^{s} \vartheta_{-t} \omega\right)\right\|_{\mathscr{C}}^{2}+\left\|z^{s}\left(\vartheta_{-t} \omega\right)\right\|_{\mathscr{C}}^{2}\right] d s \\
\leq & 4 v l_{f}^{2}\left(p_{2}(\omega)\right) \sup _{\sigma \in[-v, 0]}\left\{r_{1}\left(\vartheta_{\sigma} \omega\right)+\left\|z\left(0, \vartheta_{\sigma} \omega\right)\right\|^{2}\right\} \\
& +\sup _{\sigma \in[-v, 0]} r_{4}\left(\vartheta_{\sigma} \omega\right)+2 \nu\|g\|^{2}:=r_{5}(\omega),
\end{aligned}
$$

where

$$
p_{2}(\omega)=\sup _{\sigma \in[-\nu, 0]}\left\{\sqrt{r_{1}\left(\vartheta_{\sigma} \omega\right)}+\left\|z\left(0, \vartheta_{\sigma} \omega\right)\right\|\right\}
$$

is tempered by Remark 6. Similar to $r_{4}, r_{5}$ is also tempered. Then the lemma follows from (94) and (96).

Lemma 16. Let $B \in \mathscr{D}$ and $v^{0}(\omega) \in B(\omega)$. Then for every $\varepsilon>0$ and $\mathbb{P}$ a.e. $\omega \in \Omega$, there exist $T^{*}=T^{*}(B, \omega, \varepsilon)>0$ and $R^{*}=R^{*}(\omega, \varepsilon)>0$ such that the solution $v\left(t, \omega, v^{0}(\omega)\right)$ of problem (27)-(28) satisfies, for all $t \geq T^{*}$,

$$
\sup _{s \in[-v, 0]} \int_{|x| \geq R^{*}}\left|v^{t}\left(s, \vartheta_{-t} \omega, v^{0}\left(\vartheta_{-t} \omega\right)\right)(x)\right|^{2} d x \leq \varepsilon
$$

Proof. Let $\rho$ be a smooth function defined on $\mathbb{R}^{+}$such that $0 \leq \rho(s) \leq 1$ for all $s \geq 0$, and

$$
\rho(s)= \begin{cases}0, & 0 \leq s \leq 1, \\ 1, & s \geq 2 .\end{cases}
$$

Then there exists a positive deterministic constant $c_{2}$ such that $\left|\rho^{\prime}(s)\right| \leq c_{2}$ for all $s \geq 0$. Taking the inner product of (27) with $\rho\left(|x|^{2} / k^{2}\right) v$ in $L^{2}\left(\mathbb{R}^{d}\right)$, we get that

$$
\begin{aligned}
\frac{1}{2} & \frac{d}{d t} \int_{\mathbb{R}^{d}} \rho\left(\frac{|x|^{2}}{k^{2}}\right)|v|^{2} d x+\lambda \int_{\mathbb{R}^{d}} \rho\left(\frac{|x|^{2}}{k^{2}}\right)|v|^{2} d x \\
& -\int_{\mathbb{R}^{d}} \Delta v \rho\left(\frac{|x|^{2}}{k^{2}}\right) v d x \\
= & \int_{\mathbb{R}^{d}} f\left(v^{t}+z^{t}\right) \rho\left(\frac{|x|^{2}}{k^{2}}\right) v d x+\int_{\mathbb{R}^{d}} g \rho\left(\frac{|x|^{2}}{k^{2}}\right) v d x .
\end{aligned}
$$


We now estimate the terms in (100). First, we have that

$$
\begin{aligned}
& -\int_{\mathbb{R}^{d}} \Delta v \rho\left(\frac{|x|^{2}}{k^{2}}\right) v d x \\
& =\int_{\mathbb{R}^{d}}|\nabla v|^{2} \rho\left(\frac{|x|^{2}}{k^{2}}\right) d x+\int_{\mathbb{R}^{d}} v \rho^{\prime}\left(\frac{|x|^{2}}{k^{2}}\right) \frac{2 x}{k^{2}} \cdot \nabla v d x \\
& =\int_{\mathbb{R}^{d}}|\nabla v|^{2} \rho\left(\frac{|x|^{2}}{k^{2}}\right) d x \\
& \quad+\int_{k \leq|x| \leq \sqrt{2} k} v \rho^{\prime}\left(\frac{|x|^{2}}{k^{2}}\right) \frac{2 x}{k^{2}} \cdot \nabla v d x .
\end{aligned}
$$

Note that the second term on the right-hand side of (101) is bounded by

$$
\begin{aligned}
& \left|\int_{k \leq|x| \leq \sqrt{2} k} v \rho^{\prime}\left(\frac{|x|^{2}}{k^{2}}\right) \frac{2 x}{k^{2}} \cdot \nabla v d x\right| \\
& \quad \leq \frac{2 \sqrt{2}}{k} \int_{k \leq|x| \leq \sqrt{2} k}|v|\left|\rho^{\prime}\left(\frac{|x|^{2}}{k^{2}}\right)\right||\nabla v| d x \\
& \quad \leq \frac{4 c_{2}}{k} \int_{k \leq|x| \leq \sqrt{2} k}|v||\nabla v| d x \leq \frac{4 c_{2}}{k}\left(\|v\|^{2}+\|\nabla v\|^{2}\right) .
\end{aligned}
$$

By (101) and (102), we find that

$$
\begin{aligned}
&-\int_{\mathbb{R}^{d}} \Delta v \rho\left(\frac{|x|^{2}}{k^{2}}\right) v d x \\
& \geq \int_{\mathbb{R}^{d}}|\nabla v|^{2} \rho\left(\frac{|x|^{2}}{k^{2}}\right) d x-\frac{4 c_{2}}{k}\left(\|v\|^{2}+\|\nabla v\|^{2}\right) .
\end{aligned}
$$

For the right-hand side of (100), applying the Young inequality, we obtain that

$$
\begin{aligned}
\int_{\mathbb{R}^{d}} f\left(v^{t}+z^{t}\right) \rho\left(\frac{|x|^{2}}{k^{2}}\right) v d x & \\
\leq & \frac{c_{f}}{2} \int_{\mathbb{R}^{d}} \rho\left(\frac{|x|^{2}}{k^{2}}\right)|v|^{2} d x \\
& +\frac{1}{2 c_{f}} \int_{\mathbb{R}^{d}} \rho\left(\frac{|x|^{2}}{k^{2}}\right)\left|f\left(v^{t}+z^{t}\right)\right|^{2} d x \\
\int_{\mathbb{R}^{d}} g \rho\left(\frac{|x|^{2}}{k^{2}}\right) v d x & \leq \frac{1}{2 \beta} \int_{\mathbb{R}^{d}} g^{2} \rho\left(\frac{|x|^{2}}{k^{2}}\right) d x+\frac{\beta}{2} \int_{\mathbb{R}^{d}} \rho\left(\frac{|x|^{2}}{k^{2}}\right)|v|^{2} d x .
\end{aligned}
$$

Then it follows from (100), (103), and (104) that

$$
\begin{aligned}
\frac{d}{d t} \int_{\mathbb{R}^{d}} \rho\left(\frac{|x|^{2}}{k^{2}}\right)|v|^{2} d x & \\
\leq & -\left(2 \lambda-c_{f}-\beta\right) \int_{\mathbb{R}^{d}} \rho\left(\frac{|x|^{2}}{k^{2}}\right)|v|^{2} d x \\
& +\frac{1}{c_{f}} \int_{\mathbb{R}^{d}} \rho\left(\frac{|x|^{2}}{k^{2}}\right)\left|f\left(v^{t}+z^{t}\right)\right|^{2} d x \\
& +\frac{1}{\beta} \int_{\mathbb{R}^{d}} g^{2} \rho\left(\frac{|x|^{2}}{k^{2}}\right) d x+\frac{8 c_{2}}{k}\left(\|v\|^{2}+\|\nabla v\|^{2}\right) .
\end{aligned}
$$

Consequently,

$$
\begin{aligned}
& \frac{d}{d t}\left(e^{\alpha t} \int_{\mathbb{R}^{d}} \rho\left(\frac{|x|^{2}}{k^{2}}\right)|v|^{2} d x\right) \\
& \leq-\left(2 \lambda-c_{f}-\alpha-\beta\right) e^{\alpha t} \int_{\mathbb{R}^{d}} \rho\left(\frac{|x|^{2}}{k^{2}}\right)|v|^{2} d x \\
&+\frac{1}{c_{f}} e^{\alpha t} \int_{\mathbb{R}^{d}} \rho\left(\frac{|x|^{2}}{k^{2}}\right)\left|f\left(v^{t}+z^{t}\right)\right|^{2} d x \\
&+\frac{1}{\beta} e^{\alpha t} \int_{\mathbb{R}^{d}} g^{2} \rho\left(\frac{|x|^{2}}{k^{2}}\right) d x+\frac{8 c_{2}}{k} e^{\alpha t}\left(\|v\|^{2}+\|\nabla v\|^{2}\right) .
\end{aligned}
$$

Take $T_{1}=T_{1}(B, \omega) \geq T_{B}(\omega)+v+1$. For all $t>T_{1}$, integrating (106) over the interval $\left[T_{1}, t\right]$ leads to

$$
\begin{aligned}
& e^{\alpha t} \int_{\mathbb{R}^{d}} \rho\left(\frac{|x|^{2}}{k^{2}}\right)|v(t)|^{2} d x-e^{\alpha T_{1}} \int_{\mathbb{R}^{d}} \rho\left(\frac{|x|^{2}}{k^{2}}\right)\left|v\left(T_{1}\right)\right|^{2} d x \\
& \leq-\left(2 \lambda-c_{f}-\alpha-\beta\right) \int_{T_{1}}^{t} e^{\alpha s} \int_{\mathbb{R}^{d}} \rho\left(\frac{|x|^{2}}{k^{2}}\right)|v(s)|^{2} d x d s \\
&+\frac{1}{c_{f}} \int_{T_{1}}^{t} e^{\alpha s} \int_{\mathbb{R}^{d}} \rho\left(\frac{|x|^{2}}{k^{2}}\right)\left|f\left(v^{s}+z^{s}\right)\right|^{2} d x d s \\
&+\frac{1}{\beta} \int_{T_{1}}^{t} e^{\alpha s} \int_{\mathbb{R}^{d}} g^{2} \rho\left(\frac{|x|^{2}}{k^{2}}\right) d x d s \\
&+\frac{8 c_{2}}{k} \int_{T_{1}}^{t} e^{\alpha s}\left(\|v\|^{2}+\|\nabla v\|^{2}\right) d s .
\end{aligned}
$$

Using the Young inequality and $\left(\mathrm{A}_{3}\right)$, we obtain that

$$
\begin{aligned}
& \frac{1}{c_{f}} \int_{T_{1}}^{t} e^{\alpha s} \int_{\mathbb{R}^{d}} \rho\left(\frac{|x|^{2}}{k^{2}}\right)\left|f\left(v^{t}+z^{t}\right)\right|^{2} d x d s \\
& \quad \leq c_{f} \int_{T_{1}-v}^{t} e^{\alpha s} \int_{\mathbb{R}^{d}} \rho\left(\frac{|x|^{2}}{k^{2}}\right)|v(s)+z(s)|^{2} d x d s \\
& \leq c_{f} \int_{T_{1}}^{t} e^{\alpha s} \int_{\mathbb{R}^{d}} \rho\left(\frac{|x|^{2}}{k^{2}}\right)\left[(1+\gamma)|v(s)|^{2}\right. \\
& \quad+2 c_{f} \int_{T_{1}-v}^{T_{1}} e^{\alpha s} \int_{\mathbb{R}^{d}} \rho\left(\frac{|x|^{2}}{k^{2}}\right)\left(|v(s)|^{2}+|z(s)|^{2}\right) d x d s .
\end{aligned}
$$


By (107) and (108), we find that

$$
\begin{aligned}
& e^{\alpha t} \int_{\mathbb{R}^{d}} \rho\left(\frac{|x|^{2}}{k^{2}}\right)|v(t)|^{2} d x-e^{\alpha T_{1}} \int_{\mathbb{R}^{d}} \rho\left(\frac{|x|^{2}}{k^{2}}\right)\left|v\left(T_{1}\right)\right|^{2} d x \\
& \leq-\left(2 \lambda-(2+\gamma) c_{f}-\alpha-\beta\right) \\
& \times \int_{T_{1}}^{t} e^{\alpha s} \int_{\mathbb{R}^{d}} \rho\left(\frac{|x|^{2}}{k^{2}}\right)|v(s)|^{2} d x d s \\
&+2 c_{f} \int_{T_{1}-v}^{T_{1}} e^{\alpha s} \int_{\mathbb{R}^{d}} \rho\left(\frac{|x|^{2}}{k^{2}}\right)\left(|v(s)|^{2}+|z(s)|^{2}\right) d x d s \\
&+c_{1} \int_{T_{1}}^{t} e^{\alpha s} \int_{\mathbb{R}^{d}} \rho\left(\frac{|x|^{2}}{k^{2}}\right)|z(s)|^{2} d x d s \\
&+\frac{e^{\alpha t}}{\alpha \beta} \int_{\mathbb{R}^{d}} \rho\left(\frac{|x|^{2}}{k^{2}}\right) g^{2} d x \\
&+\frac{8 c_{2}}{k} \int_{T_{1}}^{t} e^{\alpha s}\left(\|v\|^{2}+\|\nabla v\|^{2}\right) d s \\
&+\frac{8 c_{2}}{k} \int_{T_{1}}^{t} e^{\alpha s}\left(\|v\|^{2}+\|\nabla v\|^{2}\right) d s . \\
& 2 c_{f} \int_{T_{1}-v}^{T_{1}} e^{\alpha s} \int_{\mathbb{R}^{d}} \rho\left(\frac{|x|^{2}}{k^{2}}\right)\left(|v(s)|^{2}+|z(s)|^{2}\right) d x d s \\
&+c_{1} \int_{T_{1}}^{t} e^{\alpha s} \int_{\mathbb{R}^{d}} \rho\left(\frac{|x|^{2}}{k^{2}}\right)|z(s)|^{2} d x d s \\
& \frac{2 e^{\alpha t}}{\alpha \beta} \int_{\mathbb{R}^{d}} \rho\left(\frac{|x|^{2}}{k^{2}}\right) g^{2} d x
\end{aligned}
$$

Then we have, for all $t>T_{1}$,

$$
\begin{aligned}
\int_{\mathbb{R}^{d}} \rho\left(\frac{|x|^{2}}{k^{2}}\right)|v(t)|^{2} d x \\
\leq\left[\left(1+2 v c_{f}\right)\left\|v^{T_{1}}(\omega)\right\|_{\mathscr{C}}^{2}+2 v c_{f}\left\|z^{T_{1}}(\omega)\right\|_{\mathscr{C}}^{2}\right] e^{\alpha\left(T_{1}-t\right)} \\
\quad+c_{1} \int_{T_{1}}^{t} e^{\alpha(s-t)} \int_{\mathbb{R}^{d}} \rho\left(\frac{|x|^{2}}{k^{2}}\right)|z(s)|^{2} d x d s \\
+\frac{1}{\alpha \beta} \int_{\mathbb{R}^{d}} \rho\left(\frac{|x|^{2}}{k^{2}}\right) g^{2} d x \\
+\frac{8 c_{2}}{k} \int_{T_{1}}^{t} e^{\alpha(s-t)}\left(\|v\|^{2}+\|\nabla v\|^{2}\right) d s .
\end{aligned}
$$

If we take $t \geq T_{1}+\nu$, then by (110) we find that, for all $\sigma \in$ $[-v, 0]$,

$$
\begin{aligned}
\int_{\mathbb{R}^{d}} \rho\left(\frac{|x|^{2}}{k^{2}}\right)|v(t+\sigma)|^{2} d x \\
\leq \\
\leq\left[\left(1+2 v c_{f}\right)\left\|v^{T_{1}}(\omega)\right\|_{\mathscr{C}}^{2}+2 v c_{f}\left\|z^{T_{1}}(\omega)\right\|_{\mathscr{C}}^{2}\right] e^{\alpha\left(T_{1}-t-\sigma\right)} \\
\quad+c_{1} \int_{T_{1}}^{t+\sigma} e^{\alpha(s-t-\sigma)} \int_{\mathbb{R}^{d}} \rho\left(\frac{|x|^{2}}{k^{2}}\right)|z(s)|^{2} d x d s
\end{aligned}
$$

$$
\begin{aligned}
& +\frac{1}{\alpha \beta} \int_{\mathbb{R}^{d}} \rho\left(\frac{|x|^{2}}{k^{2}}\right) g^{2} d x \\
& +\frac{8 c_{2}}{k} \int_{T_{1}}^{t+\sigma} e^{\alpha(s-t-\sigma)}\left(\|v\|^{2}+\|\nabla v\|^{2}\right) d s \\
& \leq\left[\left(1+2 v c_{f}\right)\left\|v^{T_{1}}(\omega)\right\|_{\mathscr{C}}^{2}+2 v c_{f}\left\|z^{T_{1}}(\omega)\right\|_{\mathscr{C}}^{2}\right] e^{\alpha\left(T_{1}+v-t\right)} \\
& +c_{1} e^{\alpha \nu} \int_{T_{1}}^{t} e^{\alpha(s-t)} \int_{\mathbb{R}^{d}} \rho\left(\frac{|x|^{2}}{k^{2}}\right)|z(s)|^{2} d x d s \\
& +\frac{1}{\alpha \beta} \int_{\mathbb{R}^{d}} \rho\left(\frac{|x|^{2}}{k^{2}}\right) g^{2} d x \\
& +\frac{8 c_{2}}{k} e^{\alpha \nu} \int_{T_{1}}^{t} e^{\alpha(s-t)}\left(\|v\|^{2}+\|\nabla v\|^{2}\right) d s .
\end{aligned}
$$

Then we have, for all $t \geq T_{1}+v$,

$$
\begin{aligned}
& \sup _{\sigma \in[-\nu, 0]} \int_{\mathbb{R}^{d}} \rho\left(\frac{|x|^{2}}{k^{2}}\right)\left|v^{t}(\sigma)\right|^{2} d x \\
& \leq\left[\left(1+2 \nu c_{f}\right)\left\|v^{T_{1}}(\omega)\right\|_{\mathscr{C}}^{2}+2 v c_{f}\left\|z^{T_{1}}(\omega)\right\|_{\mathscr{C}}^{2}\right] e^{\alpha\left(T_{1}+v-t\right)} \\
& +c_{1} e^{\alpha \nu} \int_{T_{1}}^{t} e^{\alpha(s-t)} \int_{\mathbb{R}^{d}} \rho\left(\frac{|x|^{2}}{k^{2}}\right)|z(s)|^{2} d x d s \\
& +\frac{1}{\alpha \beta} \int_{\mathbb{R}^{d}} \rho\left(\frac{|x|^{2}}{k^{2}}\right) g^{2} d x \\
& +\frac{8 c_{2}}{k} e^{\alpha \nu} \int_{T_{1}}^{t} e^{\alpha(s-t)}\left(\|v\|^{2}+\|\nabla v\|^{2}\right) d s .
\end{aligned}
$$

Replacing $\omega$ with $\vartheta_{-t} \omega$, we find that

$$
\begin{aligned}
\sup _{\sigma \in[-\nu, 0]} & \int_{\mathbb{R}^{d}} \rho\left(\frac{|x|^{2}}{k^{2}}\right)\left|v^{t}\left(\sigma, \vartheta_{-t} \omega, v^{0}\left(\vartheta_{-t} \omega\right)\right)\right|^{2} d x \\
\leq & {\left[\left(1+2 v c_{f}\right)\left\|v^{T_{1}}\left(\vartheta_{-t} \omega\right)\right\|_{\mathscr{C}}^{2}\right.} \\
& \left.+2 v c_{f}\left\|z^{T_{1}}\left(\vartheta_{-t} \omega\right)\right\|_{\mathscr{C}}^{2}\right] e^{\alpha\left(T_{1}+\nu-t\right)} \\
+ & c_{1} e^{\alpha \nu} \int_{T_{1}}^{t} e^{\alpha(s-t)} \int_{\mathbb{R}^{d}} \rho\left(\frac{|x|^{2}}{k^{2}}\right)\left|z\left(0, \vartheta_{s-t} \omega\right)\right|^{2} d x d s \\
+ & \frac{8 c_{2}}{k} e^{\alpha \nu} \int_{T_{1}}^{t} e^{\alpha(s-t)}\left(\left\|v\left(s, \vartheta_{-t} \omega\right)\right\|^{2}\right. \\
+ & \frac{1}{\alpha \beta} \int_{\mathbb{R}^{d}} \rho\left(\frac{|x|^{2}}{k^{2}}\right) g^{2} d x .
\end{aligned}
$$


We now estimate the terms in (113) as follows. First, replacing $t$ with $T_{1}$ and then replacing $\omega$ with $\vartheta_{T_{1}-t} \omega$ in (67), we find that

$$
\begin{aligned}
\left\|v^{T_{1}}\left(\vartheta_{-t} \omega, v^{0}\left(\vartheta_{-t} \omega\right)\right)\right\|_{\mathscr{C}}^{2} \\
\leq\left[\left(1+2 v c_{f}\right)\left\|v^{0}\left(\vartheta_{-t} \omega\right)\right\|_{\mathscr{C}}^{2}+2 v c_{f}\left\|z^{0}\left(\vartheta_{-t} \omega\right)\right\|_{\mathscr{C}}^{2}\right] e^{\alpha\left(\nu-T_{1}\right) t} \\
\quad+c_{1} e^{\alpha \nu} r\left(\vartheta_{T_{1}-t} \omega\right)+\frac{\|g\|^{2}}{\alpha \beta} .
\end{aligned}
$$

Thus,

$$
\begin{aligned}
& {\left[\left(1+2 \nu c_{f}\right)\left\|v^{T_{1}}\left(\vartheta_{-t} \omega\right)\right\|_{\mathscr{C}}^{2}+2 \nu c_{f}\left\|z^{T_{1}}\left(\vartheta_{-t} \omega\right)\right\|_{\mathscr{C}}^{2}\right] e^{\alpha\left(T_{1}+\nu-t\right)}} \\
& \leq\left(1+2 \nu c_{f}\right)\left[\left(1+2 \nu c_{f}\right)\left\|v^{0}\left(\vartheta_{-t} \omega\right)\right\|_{\mathscr{C}}^{2}\right. \\
& \left.+2 \nu c_{f}\left\|z^{0}\left(\vartheta_{-t} \omega\right)\right\|_{\mathscr{C}}^{2}\right] e^{\alpha(2 \nu-t)} \\
& +\left(1+2 \nu c_{f}\right) \frac{\|g\|^{2}}{\alpha \beta} e^{\alpha\left(T_{1}+\nu-t\right)} \\
& +c_{1}\left(1+2 \nu c_{f}\right) e^{\alpha \nu} r\left(\vartheta_{T_{1}-t} \omega\right) e^{\alpha\left(2 \nu+T_{1}-t\right)} \\
& +2 \nu c_{f}\left\|z^{0}\left(\vartheta_{T_{1}-t} \omega\right)\right\|_{\mathscr{C}}^{2} e^{\alpha\left(T_{1}+\nu-t\right)}
\end{aligned}
$$

Since $r(\omega)$ and $\left\|z^{0}(\omega)\right\|_{\mathscr{C}}^{2}$ are tempered functions, $B \in \mathscr{D}$ is tempered set and $v^{0}\left(\vartheta_{-t} \omega\right) \in B\left(\vartheta_{-t} \omega\right)$; we find from (115) that, for every $\varepsilon>0$, there exists $T_{2}=T_{2}(B, \omega, \varepsilon) \geq T_{1}+v$ such that, for all $t \geq T_{2}$,

$$
\begin{aligned}
& {\left[\left(1+2 v c_{f}\right)\left\|v^{T_{1}}\left(\vartheta_{-t} \omega\right)\right\|_{\mathscr{C}}^{2}+2 v c_{f}\left\|z^{T_{1}}\left(\vartheta_{-t} \omega\right)\right\|_{\mathscr{C}}^{2}\right] e^{\alpha\left(T_{1}+\nu-t\right)}} \\
& \quad \leq \frac{\varepsilon}{4}
\end{aligned}
$$

Next, note that

$$
\begin{aligned}
& \int_{-\infty}^{0} e^{\alpha s}\left\|z\left(0, \vartheta_{s} \omega\right)\right\|^{2} d s \\
& \quad=\int_{-\infty}^{0} e^{\alpha s} \int_{\mathbb{R}^{d}}\left|z\left(0, \vartheta_{s} \omega\right)\right|^{2} d x d s<\infty
\end{aligned}
$$

By the Lebesgue theorem of dominated convergence, there exists $R_{1}=R_{1}(\omega, \varepsilon)>0$ such that, for all $k \geq R_{1}$,

$$
\int_{-\infty}^{0} e^{\alpha s} \int_{|x| \geq k}\left|z\left(0, \vartheta_{s} \omega\right)\right|^{2} d x d s \leq \frac{\varepsilon}{4 c_{1} e^{\alpha \nu}}
$$

Then it follows from (118) that, for all $t \geq T_{2}$ and $k \geq R_{1}$,

$$
\begin{aligned}
& \int_{T_{1}}^{t} e^{\alpha(s-t)} \int_{\mathbb{R}^{d}} \rho\left(\frac{|x|^{2}}{k^{2}}\right)\left|z\left(0, \vartheta_{s-t} \omega\right)\right|^{2} d x d s \\
& \leq \int_{-\infty}^{0} e^{\alpha s} \int_{\mathbb{R}^{d}} \rho\left(\frac{|x|^{2}}{k^{2}}\right)\left|z\left(0, \vartheta_{s} \omega\right)\right|^{2} d x d s \\
& \leq \int_{-\infty}^{0} e^{\alpha s} \int_{|x| \geq k}\left|z\left(0, \vartheta_{s} \omega\right)\right|^{2} d x d s \\
& \leq \frac{\varepsilon}{4 c_{1} e^{\alpha \nu}} .
\end{aligned}
$$

For the third term on the right-side of (113), we get from (70) and (94) that, for all $t \geq T_{B}(\omega)+v+1$,

$$
\begin{aligned}
& \left\|v\left(t, \vartheta_{-t} \omega\right)\right\|^{2}+\left\|\nabla v\left(t, \vartheta_{-t} \omega\right)\right\|^{2} \\
& \quad \leq r_{5}(\omega)+\left(c_{1}+1\right) r(\omega)+\frac{\|g\|^{2}}{\alpha \beta}+1:=r_{6}(\omega) .
\end{aligned}
$$

Then by (120), we obtain that

$$
\begin{gathered}
\int_{T_{1}}^{t} e^{\alpha(s-t)}\left(\left\|v\left(s, \vartheta_{-t} \omega\right)\right\|^{2}+\left\|\nabla v\left(s, \vartheta_{-t} \omega\right)\right\|^{2}\right) d s \\
\leq \int_{T_{1}}^{t} e^{\alpha(s-t)} r_{6}\left(\vartheta_{s-t} \omega\right) d s \leq \int_{-\infty}^{0} e^{\alpha s} r_{6}\left(\vartheta_{s} \omega\right) d s .
\end{gathered}
$$

Since $r_{6}$ is tempered, the last integral in (121) exists. Therefore, there exists $R_{2}=R_{2}(\omega, \varepsilon)>0$ such that, for all $k \geq R_{2}$,

$$
\frac{8 c_{2}}{k} e^{\alpha \nu} \int_{T_{1}}^{t} e^{\alpha(s-t)}\left(\left\|v\left(s, \vartheta_{-t} \omega\right)\right\|^{2}+\left\|\nabla v\left(s, \vartheta_{-t} \omega\right)\right\|^{2}\right) d s \leq \frac{\varepsilon}{4} .
$$

Finally, since $g \in L^{2}\left(\mathbb{R}^{d}\right)$, there exists $R_{3}=R_{3}(\omega, \varepsilon)>0$ such that, for all $k \geq R_{3}(\omega, \varepsilon)$,

$$
\frac{1}{\alpha \beta} \int_{\mathbb{R}^{d}} \rho\left(\frac{|x|^{2}}{k^{2}}\right) g^{2} d x \leq \frac{1}{\alpha \beta} \int_{|x| \geq k} g^{2} d x \leq \frac{\varepsilon}{4} .
$$

Taking $R_{4}=R_{4}(\omega, \varepsilon)=\max \left\{R_{1}, R_{2}, R_{3}\right\}$, it follows from (116), (119), (122), and (123) that, for all $t \geq T_{2}$ and $k \geq R_{4}$,

$$
\begin{aligned}
& \sup _{\sigma \in[-v, 0]} \int_{|x| \geq \sqrt{2} k}\left|v^{t}\left(\sigma, \vartheta_{-t} \omega, v^{0}\left(\vartheta_{-t} \omega\right)\right)(x)\right|^{2} d x \\
& \quad \leq \sup _{\sigma \in[-v, 0]} \int_{\mathbb{R}^{d}} \rho\left(\frac{|x|^{2}}{k^{2}}\right)\left|v^{t}\left(\sigma, \vartheta_{-t} \omega, v^{0}\left(\vartheta_{-t} \omega\right)\right)\right|^{2} d x \leq \varepsilon,
\end{aligned}
$$

which completes the lemma.

Lemma 17. Let $B \in \mathscr{D}$ and $v^{0}(\omega) \in B(\omega)$. Then the solution $v\left(t, \omega, v^{0}(\omega)\right)$ of problem (27)-(28) satisfies, for all $t \geq T_{B}(\omega)+$ $\nu+1$ and $\sigma, \tau_{1}, \tau_{2} \in[-\nu, 0]$,

$$
\begin{gathered}
\left\|v^{t}\left(\sigma, \vartheta_{-t} \omega, v^{0}\left(\vartheta_{-t} \omega\right)\right)\right\|_{H^{1}\left(\mathbb{R}^{d}\right)}^{2} \leq r_{7}(\omega), \\
\left\|v^{t}\left(\tau_{1}, \vartheta_{-t} \omega, v^{0}\left(\vartheta_{-t} \omega\right)\right)-v^{t}\left(\tau_{2}, \vartheta_{-t} \omega, v^{0}\left(\vartheta_{-t} \omega\right)\right)\right\| \\
\leq r_{8}(\omega)\left|\tau_{1}-\tau_{2}\right|^{1 / 2},
\end{gathered}
$$

where $r_{7}$ and $r_{8}$ are tempered random functions. 
Proof. By (120), we find that, for all $t \geq T_{B}(\omega)+v+1$ and $\sigma \in[-v, 0]$,

$$
\begin{aligned}
& \left\|v^{t}\left(\sigma, \vartheta_{-t} \omega, v^{0}\left(\vartheta_{-t} \omega\right)\right)\right\|_{H^{1}\left(\mathbb{R}^{d}\right)}^{2} \\
& =\left\|v\left(t+\sigma, \vartheta_{-t} \omega, v^{0}\left(\vartheta_{-t} \omega\right)\right)\right\|^{2} \\
& \quad+\left\|\nabla v\left(t+\sigma, \vartheta_{-t} \omega, v^{0}\left(\vartheta_{-t} \omega\right)\right)\right\|^{2} \\
& \leq \sup _{\sigma \in[-v, 0]} r_{6}\left(\vartheta_{\sigma} \omega\right):=r_{7}(\omega) .
\end{aligned}
$$

Next, by $\left(\mathrm{A}_{1}\right)-\left(\mathrm{A}_{2}\right)$ and Lemma 14, we find that, for all $t \geq$ $T_{B}(\omega)+\nu+1$ and $\tau_{1}, \tau_{2} \in[-\nu, 0]$ (assuming $\tau_{1} \leq \tau_{2}$ for simplicity),

$$
\begin{aligned}
& \int_{t+\tau_{1}}^{t+\tau_{2}}\left\|f\left(v^{s}\left(\vartheta_{-t} \omega\right)+z^{s}\left(\vartheta_{-t} \omega\right)\right)\right\| d s \\
& \leq l_{f}\left(p_{2}(\omega)\right) \int_{t+\tau_{1}}^{t+\tau_{2}}\left\|v^{s}\left(\vartheta_{-t} \omega\right)+z^{s}\left(\vartheta_{-t} \omega\right)\right\|_{\mathscr{C}} d s \\
& \leq l_{f}\left(p_{2}(\omega)\right) \int_{t+\tau_{1}}^{t+\tau_{2}}\left[\left\|v^{s}\left(\vartheta_{-t} \omega\right)\right\|_{\mathscr{C}}+\left\|z^{s}\left(\vartheta_{-t} \omega\right)\right\|_{\mathscr{C}}\right] d s \\
& \leq l_{f}\left(p_{2}(\omega)\right)\left[\sup _{\sigma \in[-v, 0]} \sqrt{r_{1}\left(\vartheta_{\sigma} \omega\right)}+\sup _{\sigma \in[-2 v, 0]}\left\|z\left(\vartheta_{\sigma}\right)\right\|\right] \\
& \quad \times\left|\tau_{1}-\tau_{2}\right|, \sup _{\sigma \in[-v, 0]} \sqrt{r_{1}\left(\vartheta_{\sigma} \omega\right)}\left|\tau_{1}-\tau_{2}\right| .
\end{aligned}
$$

By Lemma 15, we obtain that

$$
\begin{aligned}
& \int_{t+\tau_{1}}^{t+\tau_{2}}\left\|\Delta v\left(s, \vartheta_{-t} \omega\right)\right\| d s \\
& \quad \leq\left\{\left|\tau_{1}-\tau_{2}\right| \int_{t+\tau_{1}}^{t+\tau_{2}}\left\|\Delta v\left(s, \vartheta_{-t} \omega\right)\right\|^{2} d s\right\}^{1 / 2} \\
& \quad \leq \sqrt{r_{5}(\omega)}\left|\tau_{1}-\tau_{2}\right|^{1 / 2} .
\end{aligned}
$$

Then it follows from (127), (128), and (129) that

$$
\begin{aligned}
\| v^{t} & \left(\tau_{2}, \vartheta_{-t} \omega, v^{0}\left(\vartheta_{-t} \omega\right)\right)-v^{t}\left(\tau_{1}, \vartheta_{-t} \omega, v^{0}\left(\vartheta_{-t} \omega\right)\right) \| \\
= & \left\|v\left(t+\tau_{2}, \vartheta_{-t} \omega, v^{0}\left(\vartheta_{-t} \omega\right)\right)-v\left(t+\tau_{1}, \vartheta_{-t} \omega, v^{0}\left(\vartheta_{-t} \omega\right)\right)\right\| \\
\leq & \left\|\int_{t+\tau_{1}}^{t+\tau_{2}} v^{\prime}\left(s, \vartheta_{-t} \omega\right) d s\right\| \\
\leq & \int_{t+\tau_{1}}^{t+\tau_{2}}\left\|\Delta v\left(s, \vartheta_{-t} \omega\right)\right\| d s+\lambda \int_{t+\tau_{1}}^{t+\tau_{2}}\left\|v\left(s, \vartheta_{-t} \omega\right)\right\| d s \\
& +\int_{t+\tau_{1}}^{t+\tau_{2}}\left\|f\left(v^{s}\left(\vartheta_{-t} \omega\right)+z^{s}\left(\vartheta_{-t} \omega\right)\right)\right\| d s+\int_{t+\tau_{1}}^{t+\tau_{2}}\|g\| d s \\
\leq & r_{8}(\omega)\left|\tau_{1}-\tau_{2}\right|^{1 / 2},
\end{aligned}
$$

where

$$
\begin{aligned}
& r_{8}(\omega)= \sqrt{r_{5}(\omega)} \\
&+\sqrt{\nu}\left\{\left(l_{f}\left(p_{2}(\omega)\right)+\lambda\right) \sqrt{\sup _{\sigma \in[-\nu, 0]} r_{1}\left(\vartheta_{\sigma} \omega\right)}\right. \\
&\left.+l_{f}\left(p_{2}(\omega)\right) \sup _{\sigma \in[-2 \nu, 0]}\left|z\left(\vartheta_{\sigma} \omega\right)\right|+\|g\|\right\} .
\end{aligned}
$$

It is not difficult to see that $r_{7}$ and $r_{8}$ are tempered. The lemma follows from (127) and (131).

Lemma 18. The random dynamical system $\phi$ is $\mathscr{D}$-pullback asymptotically compact in $\mathscr{C}$; that is, for $\mathbb{P}$ a.e. $\omega \in \Omega$, the sequence $\left\{\phi\left(t_{n}, \vartheta_{-t_{n}} \omega, v_{n}^{0}\left(\vartheta_{-t_{n}} \omega\right)\right)\right\}_{n=1}^{\infty}$ has a convergent subsequence in $\mathscr{C}$ provided $t_{n} \rightarrow \infty, B \in \mathscr{D}$, and $v_{n}^{0}\left(\vartheta_{-t_{n}} \omega\right) \in$ $B\left(\vartheta_{-t_{n}} \omega\right)$.

Proof. Denote by $Q_{k}$ the set $\left\{x \in \mathbb{R}^{d}:|x|<k\right\}$ for each $k \in \mathbb{N}$. Since $t_{n} \rightarrow \infty$, there exists $n_{0}=n_{0}(B, \omega) \in \mathbb{N}$ such that $t_{n}>T_{B}(\omega)+v+1$ for all $n \geq n_{0}$. Then by Lemma 17, we find that, for all $n \geq n_{0}$ and $\sigma \in[-\nu, 0]$,

$$
\left\|v^{t_{n}}\left(\sigma, \vartheta_{-t_{n}} \omega, v^{0}\left(\vartheta_{-t_{n}} \omega\right)\right)\right\|_{H^{1}\left(Q_{k}\right)}^{2} \leq r_{7}(\omega) \text {. }
$$

By the compactness of embedding $H^{1}\left(Q_{k}\right) \hookrightarrow L^{2}\left(Q_{k}\right)$, it follows from (132) that for each $\sigma \in[-\nu, 0]$ the sequence $\left\{v^{t_{n}}\left(\sigma, \vartheta_{-t_{n}} \omega, v^{0}\left(\vartheta_{-t_{n}} \omega\right)\right)\right\}_{n=1}^{\infty}$ is relatively compact in $L^{2}\left(Q_{k}\right)$. On the other hand, by Lemma 17, we also find that, for all $n \geq n_{0}$ and $\tau_{1}, \tau_{2} \in[-v, 0]$,

$$
\begin{aligned}
& \left\|v^{t_{n}}\left(\tau_{1}, \vartheta_{-t_{n}} \omega, v^{0}\left(\vartheta_{-t_{n}} \omega\right)\right)-v^{t_{n}}\left(\tau_{2}, \vartheta_{-t_{n}} \omega, v^{0}\left(\vartheta_{-t_{n}} \omega\right)\right)\right\|_{L^{2}\left(\mathrm{Q}_{k}\right)} \\
& \quad \leq r_{8}(\omega)\left|\tau_{1}-\tau_{2}\right|^{1 / 2} .
\end{aligned}
$$

Hence, the sequence $\left\{v^{t_{n}}\left(\cdot, \vartheta_{-t_{n}} \omega, v^{0}\left(\vartheta_{-t_{n}} \omega\right)\right)\right\}_{n=1}^{\infty}$ is equicontinuous. By the Ascoli-Arzela theorem, for each $k \in \mathbb{N}$ the sequence $\left\{v^{t_{n}}\left(\cdot, \vartheta_{-t_{n}} \omega, v^{0}\left(\vartheta_{-t_{n}} \omega\right)\right)\right\}_{n=1}^{\infty}$ is relatively compact in $C\left([-v, 0] ; L^{2}\left(Q_{k}\right)\right)$. Then, by a diagonal procedure, we can extract a subsequence $\left\{t_{n_{i}}\right\}$ such that for each $k \in \mathbb{N}$, the sequence $\left\{v^{t_{n_{i}}}\left(\cdot, \vartheta_{-t_{n_{i}}} \omega, v^{0}\left(\vartheta_{-t_{n_{i}}} \omega\right)\right)\right\}_{i=1}^{\infty}$ converges to $\xi_{k}(\cdot, \omega)$ in $C\left([-v, 0] ; L^{2}\left(Q_{k}\right)\right)$.

Obviously, for fixed $\sigma \in[-\nu, 0]$ and $\omega \in \Omega, \xi_{k+1}(\sigma, \omega)(\cdot)$ coincides with $\xi_{k}(\sigma, \omega)(\cdot)$ on $Q_{k}$. Therefore, one can define unambiguously a measurable function $\xi(\sigma, \omega)(\cdot)$ by stipulating that it is equal to $\xi_{k}(\sigma, \omega)(\cdot)$ on $Q_{k}$. By Lemma 13, we obtain that, for all $\sigma \in[-v, 0], k \in \mathbb{N}$, and $i>n_{0}$,

$$
\begin{aligned}
& \int_{Q_{k}}\left|v^{t_{n_{i}}}\left(\sigma, \vartheta_{-t_{n_{i}}} \omega, v^{0}\left(\vartheta_{-t_{n_{i}}} \omega\right)\right)(x)\right|^{2} d x \\
& \leq \sup _{\sigma \in[-v, 0]} \int_{\mathbb{R}^{d}}\left|v^{t_{n_{i}}}\left(\sigma, \vartheta_{-t_{n_{i}}} \omega, u^{0}\left(\vartheta_{-t_{n_{i}}} \omega\right)\right)(x)\right|^{2} d x \\
& \leq r_{1}(\omega) .
\end{aligned}
$$


Thus,

$$
\begin{aligned}
& \int_{Q_{k}}|\xi(\sigma, \omega)(x)|^{2} d x \\
& \quad=\lim _{i \rightarrow \infty} \int_{Q_{k}}\left|v^{t_{n_{i}}}\left(\sigma, \vartheta_{-t_{n_{i}}} \omega, v^{0}\left(\vartheta_{-t_{n_{i}}} \omega\right)\right)(x)\right|^{2} d x \leq r_{1}(\omega),
\end{aligned}
$$

which implies

$$
\|\xi(\sigma, \omega)\|^{2} \leq \sup _{k \in \mathbb{N}} \int_{Q_{k}}|\xi(\sigma, \omega)(x)|^{2} d x \leq r_{1}(\omega) .
$$

Hence, $\xi(\sigma, \omega) \in L^{2}\left(\mathbb{R}^{d}\right)$.

For every $B \in \mathscr{D}$ and $\varepsilon>0$, by Lemma 16, there exist $R^{*}=R^{*}(\omega, \varepsilon)>0$ and $T^{*}=T^{*}(B, \omega, \varepsilon)>0$ such that, for all $u^{0} \in B$ and $t \geq T^{*}$,

$$
\sup _{\sigma \in[-v, 0]} \int_{|x| \geq R^{*}}\left|v^{t}\left(\sigma, \vartheta_{-t} \omega, v^{0}\left(\vartheta_{-t} \omega\right)\right)\right|^{2} d x \leq \frac{\varepsilon^{2}}{8} .
$$

Since $t_{n_{i}} \rightarrow \infty$, there exists $q_{1}=q_{1}(\omega, \varepsilon) \in \mathbb{N}$ such that $t_{n_{i}} \geq T^{*}$ for all $i>q_{1}$. Then we get from (137) that, for all $i>q_{1}$,

$$
\sup _{\sigma \in[-v, 0]} \int_{|x| \geq R^{*}}\left|v^{t_{n_{i}}}\left(\sigma, \vartheta_{-t_{n_{i}}} \omega, v^{0}\left(\vartheta_{-t_{n_{i}}} \omega\right)\right)(x)\right|^{2} d x \leq \frac{\varepsilon^{2}}{8} .
$$

Take a fixed integer $k \geq R^{*}$. Then we find from (138) that, for all integer $l>k$ and $\sigma \in[-\nu, 0]$,

$$
\begin{aligned}
& \int_{k \leq|x|<l}|\xi(\sigma, \omega)(x)|^{2} d x \\
& \quad=\lim _{i \rightarrow \infty} \int_{k \leq|x|<l}\left|v^{t_{n_{i}}}\left(\sigma, \vartheta_{-t_{n_{i}}} \omega, v^{0}\left(\vartheta_{-t_{n_{i}}} \omega\right)\right)(x)\right|^{2} d x \\
& \quad \leq \sup _{\sigma \in[-v, 0], i>q_{1}} \int_{|x| \geq k}\left|v^{t_{n_{i}}}\left(\sigma, \vartheta_{-t_{n_{i}}} \omega, v^{0}\left(\vartheta_{-t_{n_{i}}} \omega\right)\right)(x)\right|^{2} d x \\
& \quad \leq \frac{\varepsilon^{2}}{8}
\end{aligned}
$$

which implies

$$
\begin{aligned}
& \sup _{\sigma \in[-v, 0]} \int_{|x| \geq k}|\xi(\sigma, \omega)(x)|^{2} d x \\
& \quad \leq \sup _{\sigma \in[-v, 0], l>k} \int_{k \leq|x|<l}|\xi(\sigma, \omega)(x)|^{2} d x \leq \frac{\varepsilon^{2}}{8} .
\end{aligned}
$$

As the sequence $\left\{v^{t_{n_{i}}}\left(\cdot, \vartheta_{-t_{n_{i}}} \omega, v^{0}\left(\vartheta_{-t_{n_{i}}} \omega\right)\right)\right\}_{i=1}^{\infty}$ converges to $\xi(\cdot, \omega)$ in $C\left([-v, 0] ; L^{2}\left(Q_{k}\right)\right)$, there exists $q_{2}=q_{2}(\omega, \varepsilon) \in \mathbb{N}$ such that, for all $i>q_{2}$,

$$
\begin{aligned}
& \sup _{\sigma \in[-v, 0]} \int_{Q_{k}}\left|v^{t_{n_{i}}}\left(\sigma, \vartheta_{-t_{n_{i}}} \omega, v^{0}\left(\vartheta_{-t_{n_{i}}} \omega\right)\right)(x)-\xi(\sigma, \omega)(x)\right|^{2} d x \\
& \quad \leq \frac{\varepsilon^{2}}{2} .
\end{aligned}
$$

Let $q=\max \left\{q_{1}, q_{2}\right\}$. Then it follows from (138), (140), and (141) that, for all $i>q$,

$$
\begin{aligned}
& \sup _{\sigma \in[-v, 0]}\left\|v^{t_{n_{i}}}\left(\sigma, \vartheta_{-t_{n_{i}}} \omega, v^{0}\left(\vartheta_{-t_{n_{i}}} \omega\right)\right)-\xi(\sigma, \omega)\right\|^{2} \\
& \leq \sup _{\sigma \in[-v, 0]} \int_{Q_{k}} \mid u^{t_{n_{i}}}\left(\sigma, \vartheta_{-t_{n_{i}}} \omega, u^{0}\left(\vartheta_{-t_{n_{i}}} \omega\right)\right)(x) \\
& \quad-\left.\xi(\sigma, \omega)(x)\right|^{2} d x \\
& \quad+2 \sup _{\sigma \in[-v, 0]} \int_{|x| \geq k}|\xi(\sigma, \omega)(x)|^{2} d x \\
& \quad+2 \sup _{\sigma \in[-\nu, 0]} \int_{|x| \geq k}\left|v^{t_{n_{i}}}\left(\sigma, \vartheta_{-t_{n_{i}}} \omega, v^{0}\left(\vartheta_{-t_{n_{i}}} \omega\right)\right)(x)\right|^{2} d x \\
& \leq \varepsilon^{2},{ }^{2}
\end{aligned}
$$

which shows that the sequence $\left\{v^{t_{n_{i}}}\left(\cdot, \vartheta_{-t_{n_{i}}} \omega, v^{0}\left(\vartheta_{-t_{n_{i}}} \omega\right)\right)\right\}_{i=1}^{\infty}$ converges to $\xi(\cdot, \omega)$ in $\mathscr{C}$. This completes the proof.

We are now in a position to present our main result about the existence of a $\mathscr{D}$-random attractor for $\psi$ in $\mathscr{C}$.

Theorem 19. The random dynamical system $\psi$ has a unique $\mathscr{D}$-random attractor in $\mathscr{C}$.

Proof. Notice that $\phi$ has a closed absorbing set $K$ in $\mathscr{D}$ by Lemma 13 and is $\mathscr{D}$-pullback asymptotically compact in $\mathscr{C}$ by Lemma 18 . Hence, the existence of a unique $\mathscr{D}$-random attractor $\left\{\mathscr{A}_{1}(\omega)\right\}_{\omega \in \Omega}$ for $\phi$ follows from Proposition 10 immediately.

Since $\psi$ and $\phi$ are conjugated by the random homeomorphism $T(\omega, \xi)=\xi+z^{0}(\omega)$ and $z^{0}(\omega) \in \mathscr{C}$ is tempered, then, by Proposition 1.8.3 in [33], $\psi$ has a unique $\mathscr{D}$-random attractor $\left\{\mathscr{A}_{2}(\omega)\right\}_{\omega \in \Omega}$ in $\mathscr{C}$ which is given by

$$
\mathscr{A}_{2}(\omega)=\left\{\xi(\omega)+z^{0}(\omega): \xi(\omega) \in \mathscr{A}_{1}(\omega)\right\} .
$$

The proof is complete.

\section{Acknowledgments}

The authors are very grateful to the anonymous reviewers for their careful reading of the paper and valuable comments, which improved the presentation of this paper. This work is partially supported by the National Natural Science Foundation of China under Grants 11071166 and 11271110, the Key Programs for Science and Technology of the Education Department of Henan Province under Grant 12A110007, and the Scientific Research Start-up Funds of Henan University of Science and Technology.

\section{References}

[1] H. Crauel and F. Flandoli, "Attractors for random dynamical systems," Probability Theory and Related Fields, vol. 100, no. 3, pp. 365-393, 1994. 
[2] F. Flandoli and B. Schmalfuss, "Random attractors for the 3D stochastic Navier-Stokes equation with multiplicative white noise," Stochastics and Stochastics Reports, vol. 59, no. 1-2, pp. 21-45, 1996.

[3] J. K. Hale, Asymptotic Behavior of Dissipative Systems, American Mathematical Society, Providence, RI, USA, 1988.

[4] A. V. Babin and M. I. Vishik, Attractors of Evolution Equations, North-Holland, Amsterdam, The Netherlands, 1992.

[5] R. Temam, Infinite Dimensional Dynamical Systems in Mechanics and Physics, Springer, New York, NY, USA, 2nd edition, 1997.

[6] H. Crauel, A. Debussche, and F. Flandoli, "Random attractors," Journal of Dynamics and Differential Equations, vol. 9, no. 2, pp. 307-341, 1997.

[7] X. Fan, "Random attractors for damped stochastic wave equations with multiplicative noise," International Journal of Mathematics, vol. 19, no. 4, pp. 421-437, 2008.

[8] Y. Li and B. Guo, "Random attractors for quasi-continuous random dynamical systems and applications to stochastic reactiondiffusion equations," Journal of Differential Equations, vol. 245, no. 7, pp. 1775-1800, 2008.

[9] P. W. Bates, K. Lu, and B. Wang, "Random attractors for stochastic reaction-diffusion equations on unbounded domains," Journal of Differential Equations, vol. 246, no. 2, pp. 845-869, 2009.

[10] B. Wang, "Random attractors for the stochastic BenjaminBona-Mahony equation on unbounded domains," Journal of Differential Equations, vol. 246, no. 6, pp. 2506-2537, 2009.

[11] B. Wang, "Random attractors for the stochastic FitzHughNagumo system on unbounded domains," Nonlinear Analysis: Theory, Methods \& Applications, vol. 71, no. 7-8, pp. 2811-2828, 2009.

[12] Z. Shen, S. Zhou, and W. Shen, "One-dimensional random attractor and rotation number of the stochastic damped sineGordon equation," Journal of Differential Equations, vol. 248, no. 6, pp. 1432-1457, 2010.

[13] B. Wang, "Asymptotic behavior of stochastic wave equations with critical exponents on $\mathbb{R}^{3}$," Transactions of the American Mathematical Society, vol. 363, no. 7, pp. 3639-3663, 2011.

[14] B. Gess, "Random attractors for degenerate stochastic partial differential equations," Journal of Dynamics and Differential Equations, vol. 25, no. 1, pp. 121-157, 2013.

[15] B. Gess, W. Liu, and M. Röckner, "Random attractors for a class of stochastic partial differential equations driven by general additive noise," Journal of Differential Equations, vol. 251, no. 45, pp. 1225-1253, 2011.

[16] W.-J. Beyn, B. Gess, P. Lescot, and M. Röckner, "The global random attractor for a class of stochastic porous media equations," Communications in Partial Differential Equations, vol. 36, no. 3, pp. 446-469, 2011.

[17] Z. Wang, S. Zhou, and A. Gu, "Random attractor for a stochastic damped wave equation with multiplicative noise on unbounded domains," Nonlinear Analysis: Real World Applications, vol. 12, no. 6, pp. 3468-3482, 2011.

[18] Z. Wang and S. Zhou, "Random attractor for stochastic reaction-diffusion equation with multiplicative noise on unbounded domains," Journal of Mathematical Analysis and Applications, vol. 384, no. 1, pp. 160-172, 2011.

[19] W. Zhao and Y. Li, " $\left(L^{2}, L^{p}\right)$-random attractors for stochastic reaction-diffusion equation on unbounded domains," Nonlinear Analysis: Theory, Methods \& Applications, vol. 75, no. 2, pp. 485-502, 2012.
[20] A. Gu, "Random attractors of stochastic three-component reversible Gray-Scott system on unbounded domains," Abstract and Applied Analysis, vol. 2012, Article ID 419472, 22 pages, 2012.

[21] L. Wang and D. Xu, "Asymptotic behavior of a class of reactiondiffusion equations with delays," Journal of Mathematical Analysis and Applications, vol. 281, no. 2, pp. 439-453, 2003.

[22] T. Caraballo and J. Real, "Attractors for 2D-Navier-Stokes models with delays," Journal of Differential Equations, vol. 205, no. 2, pp. 271-297, 2004.

[23] J. Li and J. Huang, "Uniform attractors for non-autonomous parabolic equations with delays," Nonlinear Analysis: Theory, Methods \& Applications, vol. 71, no. 5-6, pp. 2194-2209, 2009.

[24] P. Marín-Rubio, J. Real, and J. Valero, "Pullback attractors for a two-dimensional Navier-Stokes model in an infinite delay case," Nonlinear Analysis: Theory, Methods \& Applications, vol. 74, no. 5, pp. 2012-2030, 2011.

[25] M. Conti and M. Coti Zelati, "Attractors for the Cahn-Hilliard equation with memory in 2D," Nonlinear Analysis: Theory, Methods \& Applications, vol. 72, no. 3-4, pp. 1668-1682, 2010.

[26] P. Marín-Rubio and J. Real, "Attractors for 2D-Navier-Stokes equations with delays on some unbounded domains," Nonlinear Analysis: Theory, Methods \& Applications, vol. 67, no. 10, pp. 2784-2799, 2007.

[27] I. Chueshov and M. Scheutzow, "On the structure of attractors and invariant measures for a class of monotone random systems," Dynamical Systems, vol. 19, no. 2, pp. 127-144, 2004.

[28] X. Ding and J. Jiang, "Random attractors for stochastic retarded lattice dynamical systems," Abstract and Applied Analysis, vol. 2012, Article ID 409282, 27 pages, 2012.

[29] W. Yan, Y. Li, and S. Ji, "Random attractors for first order stochastic retarded lattice dynamical systems," Journal of Mathematical Physics, vol. 51, no. 3, Article ID 032702, 17 pages, 2010.

[30] B. Wang, "Attractors for reaction-diffusion equations in unbounded domains," Physica D, vol. 128, no. 1, pp. 41-52, 1999.

[31] A. Rodriguez-Bernal and B. Wang, "Attractors for partly dissipative reaction diffusion systems in $\mathbb{R}^{n}$," Journal of Mathematical Analysis and Applications, vol. 252, no. 2, pp. 790-803, 2000.

[32] L. Arnold, Random Dynamical Systems, Springer, Berlin, Germany, 1998.

[33] I. Chueshov, Monotone Random Systems Theory and Applications, Springer, Berlin, Germany, 2002.

[34] P. W. Bates, H. Lisei, and K. Lu, "Attractors for stochastic lattice dynamical systems," Stochastics and Dynamics, vol. 6, no. 1, pp. $1-21,2006$.

[35] G. da Prato and J. Zabczyk, Stochastic Equations in Infinite Dimensions, Cambridge University Press, Cambridge, UK, 1992.

[36] A. Pazy, Semigroups of Linear Operators and Applications to Partial Differential Equations, Springer, New York, NY, USA, 1983.

[37] A. Bensoussan, G. da Prato, M. C. Delfour, and S. K. Mitter, Representation and Control of Infinite Dimensional Systems, Birkhäauser, Boston, Mass, USA, 2nd edition, 2007. 


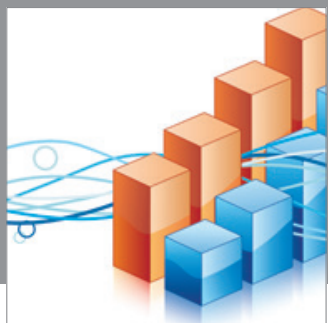

Advances in

Operations Research

mansans

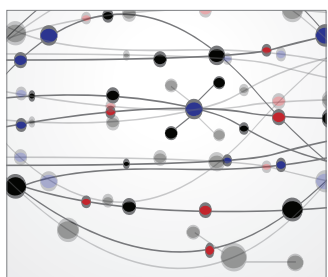

The Scientific World Journal
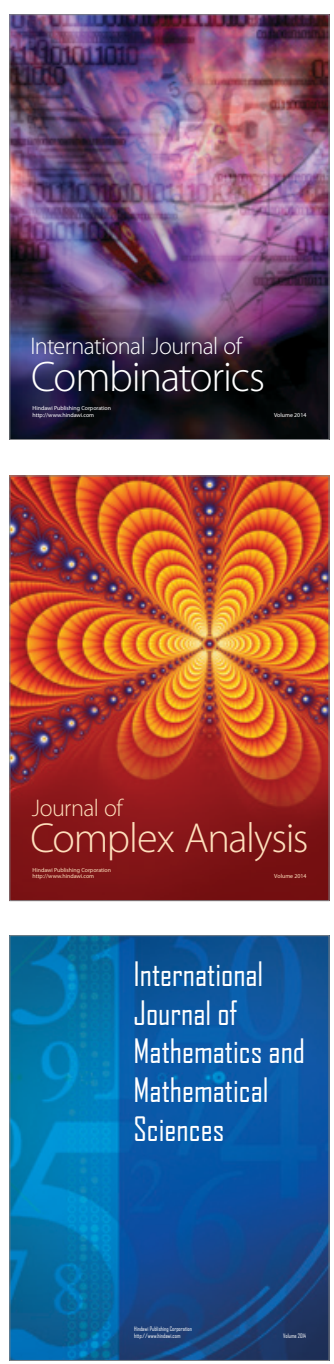
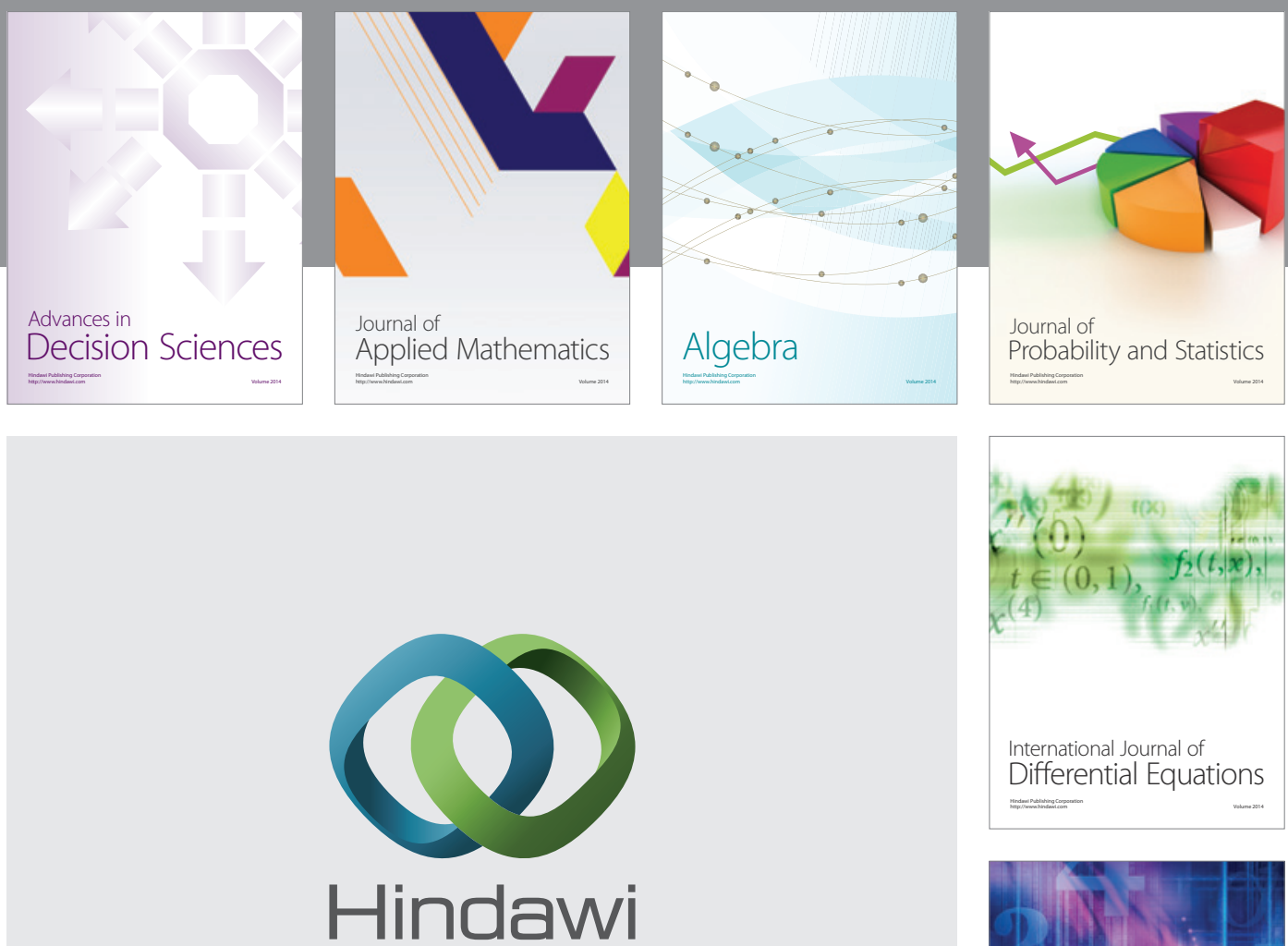

Submit your manuscripts at http://www.hindawi.com
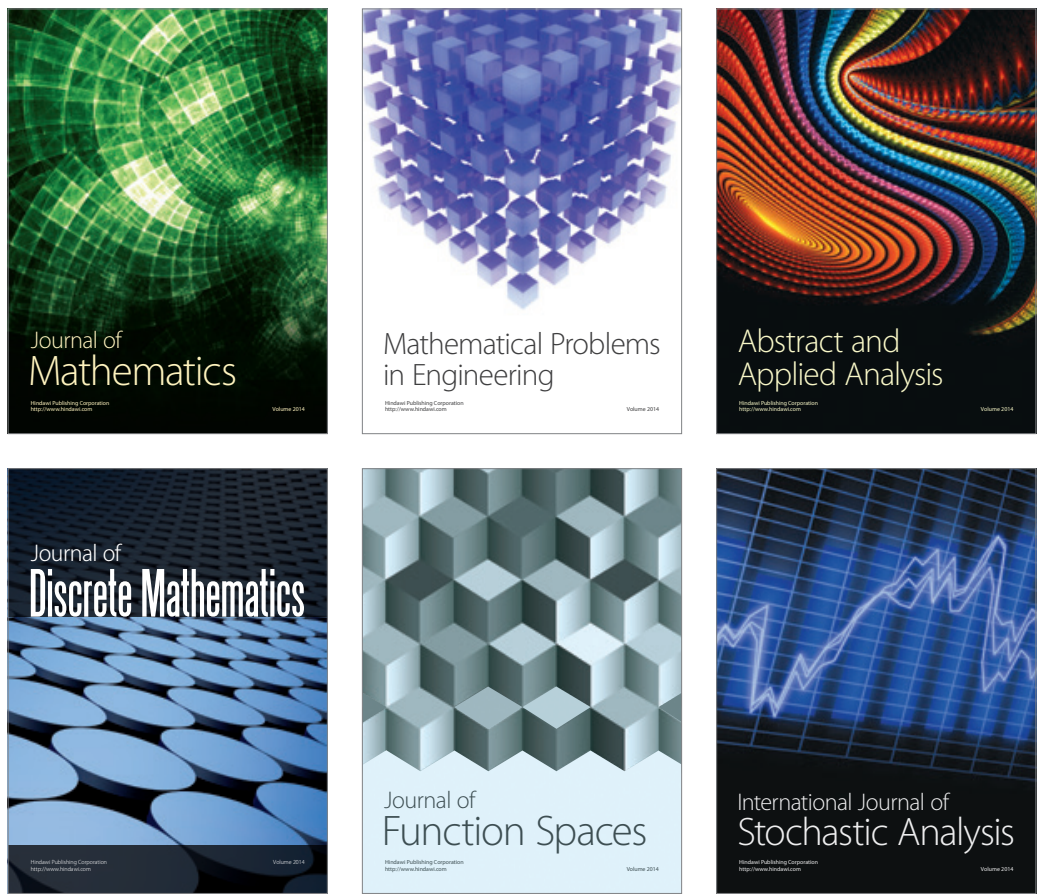

Journal of

Function Spaces

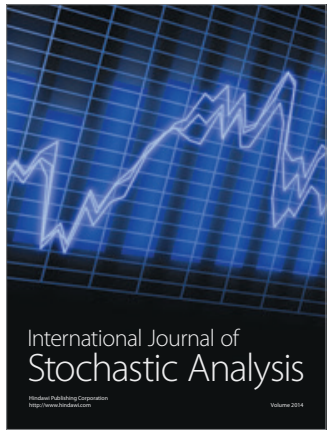

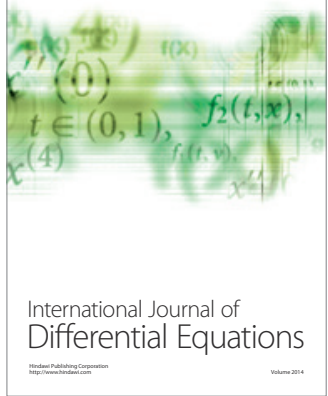
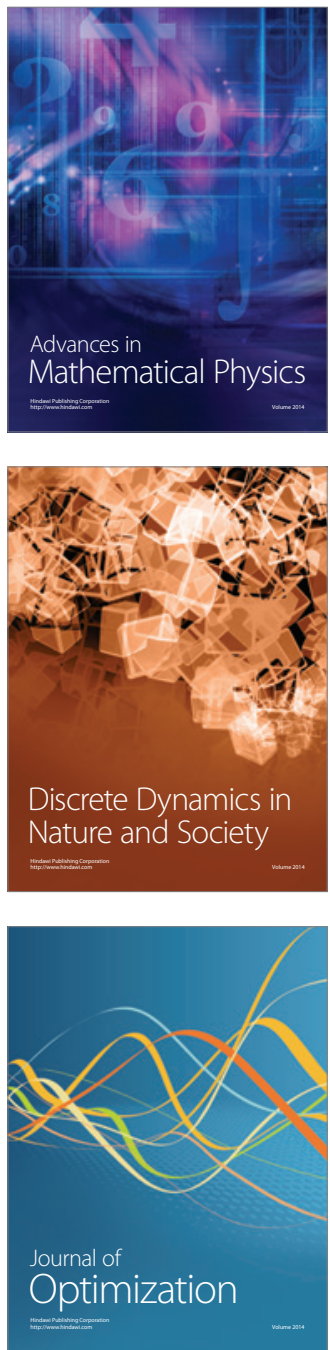